The abstracts are only available online, free of charge, at www.karger.com/doi/10.1159/000103455

\title{
27th Annual Meeting of the Swiss Society of Biological Psychiatry
}

\section{Sleep in Psychiatry - State of the Art}

Basel, March 15, 2007

President

Werner Strik, Bern

Guest Editors

Edith Holsboer-Trachsler, Basel

Martin Hatzinger, Basel

\section{Contents}

Symposium Lectures

Abstracts S1-S4

Oral Communications

Poster Presentations

Abstracts P1-P31 


\section{Symposium Lectures}

S1

\section{Psychoimmunology and Sleep}

T. Pollmächer

Center of Mental Health, Ingolstadt, Germany

Already the Greek philosopher Aristotle, in a small book on the pecularities of sleep, mentioned sleepiness as concomitant of fever, suggesting that host defense activation during infectious diseases has a profound influence on the regulation of sleep and wakefulness. In turn, in the 1980's, Pappenheimer and Krueger purified sleep-inducing factor $\mathrm{S}$ from the brains of sleep deprived animals which later was identified as muramyl peptide, a cell-wall component of gram-positive bacteria. This discovery led to the idea of a reciprocal interaction between sleep and the immune system. During the last 2 decades it has clearly been shown, first in animal and later in human studies, that stimulation of host defense mechanisms affects sleep wake behaviour through the liberation of various cytokines, which are humoral immune mediators affecting numerous brain functions. These cytokines are also involved in the pathophysiology of numerous other disorders such as cancer, autoimmune disorders and even obesity, suggesting that immune mediators might be of crucial importance for altered sleep in many medical conditions. Moreover, there is evidence that cytokines are involved in physiological sleep regulation as well. In contrast, there is much less scientific evidence regarding the question whether and how alterations in sleep wake behaviour and sleep loss in particular affect functions of the immune systems. Some studies suggest that cytokine levels and other immune tests may change with sleep loss, but the clinical significance of these findings is unclear, because it could not be shown so far that sleep deprivation alters the host response to immune challenges in vivo. To conclude, immune mediators might be of significant meaning for physiological sleep regulation and changes of sleep wake behaviour during a variety of medical disorders, but it remains unclear whether sleep, in turn, has clinically relevant effects on immune functions.

\section{S2}

\section{Mood and Sleep}

M. Hatzinger

Psychiatric Out-Patient Department, University Hospital, Basel, Switzerland

Sleep in mood disorders has been a topic of research for many years. During the past decades, the role of disturbed sleep has aroused great interest in the search for biological abnormalities especially in depression. Sleep complaints have been considered as cardinal symptoms of the depressive state for as long as this disorder has been recognized. About $90 \%$ of the patients suffering from a major depressive episode report complaints of initiating and maintaining sleep as well as early awakening and remaining awake. Moreover, depression is quite prevalent in insomniacs whereas insomnia is more than twice as prevalent in depressed than in non-depressed individuals. Sleep disturbances in depression have been documented objectively by means of polysomnography: Disturbed sleep maintenance, a deficit of slow wave sleep (SWS) especially during the first sleep cycle, an earlier onset of the first rapid eye movement (REM) sleep period (shortened REM latency), and increased density of REM during REM sleep (REM density) are well-established findings. Among these alterations a continuance of shortened REM sleep latencies during remission was found to be associated with an increased risk for relapses or recurrences in short-term follow-ups. The predictive value of these sleep alterations for long-term course of depression, so far unclear, was assessed in a long-term study over 8 years. It turned out that especially the amount of SWS during the first sleep cycle and REMdensity are of predictive value for the long-term course of major depression. Besides sleep abnormalities hypothalamic-pituitaryadrenocortical (HPA) system dysfunction is a prominent feature in the acute depressed state. Moreover, recent data have demonstrated that the occurrence and/or persistence of HPA system dysfunction in depression may also be related to an unfavorable course as reflected by relapse in short-term or recurrence in long-term outcome. Importantly, HPA system function is closely intertwined with sleep as reflected by the extended neuroendocrinological-neurophysiological two process model of sleep regulation. In the above mentioned longterm study HPA axis dysregulation was closely related with the sleep alterations predictive for long-term outcome i.e. SWS and REM density. However, most of the data focusing on these neurobiological abnormalities are from studies after the disorder's onset. Therefore, a project in pre-school children was started aiming to objectively investigate sleep, HPA axis function, and psychological/behavioural variables in order to identify risk factors early in development. The first cross-sectional results of this long-term study showed an unfavourable sleep profile associated with an increased HPA axis activity. Furthermore, bad sleep was related to more difficult behavioural/psychological dimensions. The longitudinal follow up of this sample will demonstrate if these biological abnormalities are predictive for the onset of clinically relevant psychiatric problems such as depression. In summary, considering the recent advances in the knowledge of the sleep-mood interaction it may be expected that sleep research may contribute as functional phenotyping to more comprehensive hypotheses explaining the link between sleep, the symptomatology of depression and the underlying pathophysiology of this disorder.

\begin{tabular}{ll}
\hline KARGER & ( ) 2007 S. Karger AG, Basel \\
0302-282X/07/0551-0053 \\
$\begin{array}{l}\text { Fax +4161306 1234 } \\
\begin{array}{l}\text { E-Mail karger@karger.ch } \\
\text { www.karger.com }\end{array}\end{array}$ & $\begin{array}{l}\text { Accessible online at: } \\
\text { www.karger.com/nps }\end{array}$
\end{tabular}


S3

\section{Imaging and Sleep}

H.-P. Lando/t ${ }^{1,2}$

${ }^{1}$ Institute of Pharmacology and Toxicology, University of Zürich, ${ }^{2} Z$ ürich Center for Integrative Human Physiology (ZIHP), University of Zürich, Zürich, Switzerland

The most important aspect of sleep in relation to its function is its homeostatic regulation. The time course of delta activity (spectral power with $\sim 0.75-4.5 \mathrm{~Hz}$ ) in the electroencephalogram (EEG) during sleep reliably reflects sleep homeostasis and the cyclic alternation between non-rapid-eye-movement (non-REM; high delta activity) and rapid-eye-movement (REM; low delta activity) sleep episodes. Global cerebral blood flow and metabolism decrease from wakefulness to non-REM sleep and increase to waking levels again in REM sleep $[1,2]$. In non-REM sleep the measurement of regional cerebral blood flow (rCBF) with $\mathrm{H}_{2}{ }^{15} \mathrm{O}$ positron emission tomography (PET) revealed in healthy volunteers that $\mathrm{rCBF}$ decreases as a function of increasing delta activity, particularly in prefrontal cortical areas [3, 4]. These findings are consistent with multi-channel EEG mapping showing highest delta activity in frontal EEG leads [5, 6]. Patients suffering from major depression were found to exhibit reduced sleeprelated decrease in regional cerebral glucose metabolism measured with ${ }^{18}$ F-Fluoro-deoxy-glucose PET. More specifically, the reduction in glucose metabolism from pre-sleep wakefulness to non-REM sleep was substantially less extensive in frontal regions in patients when compared to in healthy controls $[7,8]$. While only a few studies have applied EEG mapping during sleep in psychiatric populations, the available data in moderately depressed patients do not suggest impaired homeostatic sleep regulation $[9,10]$. This notion is further supported by the dynamics of delta activity after pharmacological suppression of REM sleep with a classical monoamine oxidase inhibitor [11]. We conclude that combined brain imaging and EEG mapping studies in depressed patients are needed to explore whether the distinct neuroanatomic abnormalities are related to altered dynamics of sleep homeostasis and EEG topography during sleep.

\section{References}

1. Braun, et al: Brain 1997;120:1173-1197.

2. Nofzinger, et al: Brain 2002;125:1105-1115.

3. Finelli, et al: J Sleep Res 2000;9:161-173.

4. Dang-Vu, et al: NeuroImage 2005;28:14-21.

5. Finelli, et al: Eur J Neurosci 2001;13:2282-2290.

6. Tinguely, et al: NeuroImage 2006;32:283-292.

7. Germain, et al: Am J Psychiatry 2004;161:1856-1863.

8. Nofzinger, et al: Arch Gen Psychiatry 2005;62:387-396

9. Landolt, Gillin: Sleep 2005;28:239-247.

10. Ferrarelli, et al: Am J Psychiatry 2007;164, in press.

11. Landolt, et al: Arch Gen Psychiatry 2001;58:268-276.

\section{S4}

\section{Sleep and Neurology}

\section{C.L. Bassetti}

Neurology Department, University Hospital, Zürich, Switzerland

'Sleep is by the brain and for the brain'. Sleep, an active process of the brain, plays an important role in neuronal recuperation. As a consequence sleep is frequently disturbed in the course of neurological disorders. On the other hand, sleep disorders can have detrimental effects on memory, learning and frontal lobe functions. Finally, 'dissociated states' arising from sleep disorders offer unique insights into the neurobiology of consciousness. Four clinical situations will be presented to illustrate the clinical and scientific interest that can result from the study of neurological sleep disorders: (1) Hypersomnia after thalamic stroke ('sleep and the cognitive brain'); (2) Narcolepsy with cataplexy ('sleep and the emotional brain'); (3) Sleepwalking ('sleep and the violent brain') and (4) REM sleep behaviour disorder ('sleep and the dreaming brain'). 


\section{Oral Communications}

\section{V1 \\ Grey Matter Volume Reductions in Individuals at High Risk of Psychosis}

S. Borgwardt, A. Riecher-Rössler, P. Dazzan, X. Chitnis, J. Aston, M. Drewe, U. Gschwandtner, S. Haller, M. Pflüger, E. Rechsteiner, M. D'Souza, R.-D. Stieglitz, E.W. Radü, P.K. McGuire

Psychiatric Outpatient Department, University Hospital, Basel, Switzerland

Objective: To investigate brain structure in individuals with 'prodromal' symptoms, who are at high risk of developing schizophrenia, before they develop psychosis. Methods: Magnetic resonance imaging data were acquired using a $1.5 \mathrm{~T}$ scanner from 35 individuals at high risk of developing psychosis, according to the PACE criteria (HR), 25 patients with a first-episode psychosis (FE) and 22 healthy volunteers (HC). 12 (34\%) of the HR group developed a psychotic disorder after scanning. The subjects were recruited from the Basel Early Detection of Psychosis Clinic (FEPSY). Images were processed and analysed using voxel based morphometry (VBM). Results: Individuals at high risk of psychosis (HR) showed grey matter differences in the superior temporal gyrus, cingulate, precuneus and the left insula $(\mathrm{p}<0.002)$ compared to healthy controls $(\mathrm{HC})$ and patients with a first episode psychosis (FE). Within the HR group, grey matter volume in the right insula, and the inferior and superior temporal gyrus was smaller in those who later developed psychosis than in those who did not $(p<0.002)$. Conclusions: Individuals with prodromal symptoms show regional grey matter differences relative to controls and patients with first-episode psychosis. Some of these may be a correlate of vulnerability to psychosis, while others may be particularly associated with subsequent transition to psychosis.

\section{V2}

\section{Glutathione Deficit in Schizophrenia: Replenishment of Glutathione Levels in Neurons and Astrocytes Models}

S. Lavoie, P. Steullet, R. Gysin, M. Cuenod, K. Q. Do

Center for Psychiatric Neuroscience, Department of Psychiatry, Lausanne, Switzerland

In schizophrenia patients, a decrease in glutathione levels ([GSH]) in cerebrospinal fluid and prefrontal cortex was observed. Several evidences suggest a compromised GSH synthesis at the level of the rate limiting enzyme, glutamyl-cysteine ligase (GCL): association of allelic variants of GCL modulatory subunit (GCLM) gene with the illness; decrease of mRNA levels of both GCLM and GCL catalytic subunit (GCLC) in patients fibroblasts; decrease of GCLC protein expression and of GCL activity under oxidative stress conditions. The aim of this study is to find substances that could normalize [GSH] in cultured neurons and astrocytes with compromised GCL activity. First, GCL activity was inhibited with BSO, a blocker of GCLC. In both neurons and astrocytes, the decrease in [GSH] was prevented by a membrane permeable GSH analogue, GSH-ethyl-ester, that bypasses the GCL synthesis step. As a second model, we used neurons and astrocytes from GCLM knockout $(-/-)$ mice. These cells show low $[\mathrm{GSH}](-80 \%)$ and GCL activity $(-25 \%)$. We tested natural antioxidants [Curcumin (polyphenol); quercetin (flavonoid)] and tert-butylhydroquinone (tBHQ), a quinone that generates free radicals. These substances are known for their capacity to increase [GSH] in various cell types. In wild-type $(+/+)$ astrocytes, [GSH] and GCL activity were increased by curcumin $(50 \mathrm{~mm} ; 50 \%$; $140 \%$; respectively), tBHQ (100 mM; $80 \% ; 150 \%)$, and quercetin $(20-100 \mathrm{~mm}$; $60 \% ; 100 \%$. In $(+/+)$ neurons, curcumin was also efficient $(10 \mathrm{mM}$; $60 \% ; 80 \%)$ and, while low [tBHQ] $(20 \mu \mathrm{M})$ increased [GSH] $(20 \%)$, higher [tBHQ] and [quercetin] depleted [GSH] and led to cell death. These results suggest that neurons and astrocytes differ in their ability to regulate GSH synthesis and to cope with the toxic effect of some substances. In GCLM (-/-) astrocytes, curcumin slightly increased GCL activity (20\%), while tBHQ had no effect and quercetin led to [GSH] depletion even at low concentrations. This indicates that GCLM seems to be necessary for enhancement of GSH synthesis by tBHQ, curcumin and quercetin. Furthermore, it suggests that a compromised GSH synthesis due to a defect at the level of GCL might increase brain cells sensitivity to oxidative stress and substances known to be antioxidants might become prooxidants.

\section{V3}

\section{Activation of the Primary Auditory Cortex in Hallucinating Schizophrenia Patients during Inner Speech}

\author{
D. Hubl, C. van Swam, T. Koenig, W. Strik, T. Dierks \\ Department of Psychiatric Neurophysiology, University \\ Hospital of Psychiatry, Bern, Switzerland
}

Auditory verbal hallucinations (AVH) may result from deficits in auditory self monitoring during inner speech. Thus, inner speech might be misidentified as alien and not as self generated. AVH are most common in schizophrenia and their occurence allows to diagnose schizophrenia. Main part of the neuroanatomical basis for AVH is located in the speech and language related cortex and their white matter interconnections. In detail, pathological activation during AVH have been found in the primary auditory cortex, in motor and sensory speech areas. Using 76-channel EEG, the aim of this study was to investigate the cortical activations evoked by a verbal fluency task, a task to provoke inner speech. 14 chronic schizophrenic patients with persistent AVH lasting for at least 4 years and 9 healthy controls were measured. The EEG was analysed for periods with and without task performance. First, the EEG was frequency transformed, than SLORETA current densities were computed separately for the different frequency bands. The (preliminary) statistical analysis compared current densities between patients and controls during task execution using voxel-wise t-tests and ANOVAs in the regions of interest. In the lower beta band, we found increased brain electrical activity of the primary auditory cortex in patients during inner speech, whereas 
controls showed reduced activity during task execution. These results support the hypothesis of an inhibition of the auditory system during self generated, inner speech. Interestingly, patients prone to AVH lack this inhibition and show on the contrary an (pathological) activation of the auditory cortex. This activation may 'label' self generated speech as coming from outside, and give it an alien character, a sensation which is then termed hallucination.

\section{V4}

\section{A Rat Model Suggesting that} Hypersensitivity to Corticotrophin-Releasing Hormone (CRH) May be an Endophenotype for Vulnerability to Depression

\author{
T. Steimer ${ }^{1}$, A. Python ${ }^{1}$, A. Laverrièr ${ }^{1}$, P. Schulz', \\ J.-M. Aubry ${ }^{2}$ \\ ${ }^{1}$ Psychopharmacologie clinique (APSI) and ${ }^{2}$ Dept. de \\ Psychiatrie, Programme Bipolaire, Hôpitaux Universitaires \\ de Genève (HUG), Chêne-Bourg, Switzerland
}

Aim of the Study: Two tests used to assess hypothalamopituitary-adrenal (HPA) axis dysregulation in major depressive disorder (MDD) patients, i.e. the dexamethasone (DEX) suppression test (DST), and the DEX/CRH test (DST with a CRH challenge), were applied to a putative model of genetic vulnerability to depression in rodents, the Roman high-(RHA) and low-(RLA) avoidance rat lines. RLA rats are more sensitive to stress and prone to develop depressivelike features when exposed to aversive events, as compared to RHA rats. Recent clinical data suggest that the DEX/CRH test may reveal a vulnerability (or risk factor) for affective disorders. Methods: DST: Rats were injected s.c. with dexamethasone $(30 \mu \mathrm{g} / \mathrm{kg}$ body weight) at $10.00 \mathrm{~h}$ and killed by brief $(<2 \mathrm{~min})$ exposure to $\mathrm{CO}_{2}$ between 19.00 and $21.00 \mathrm{~h}$. DEX/CRH test: rats equipped with a permanent jugular vein catheter one week before the experiment were injected i.v. with dexamethasone $(30 \mu \mathrm{g} / \mathrm{kg}$ body weight) at $10.00 \mathrm{~h}$. Three successive blood samples were collected at 15.00, 15.30, and $16.00 \mathrm{~h}$ to establish baseline hormone levels and ovine CRH (o-CRH, Clinalfa, $500 \mathrm{ng} / \mathrm{kg}$ body weight) was injected via the catheter at $16.05 \mathrm{~h}$. Further blood samples were obtained 10, 20, 40 and $60 \mathrm{~min}$ after $\mathrm{CRH}$ injection. Hormone assays: Corticosterone (CORT) was measured using an enzyme immunoassay (EIA, Assay Designs' Correlate-EIA ${ }^{\mathrm{TM}}$ ). Intra-assay variation was $<9 \%$, inter-assay variation $<12 \%$, with a sensitivity limit of about $30 \mathrm{pg} / \mathrm{mL}$. CRH receptors: those were measured using SDS-PAGE and Western blotting with a specific CRH antibody. Results: (a) in the DST, CORT was suppressed to the same extent in both lines; (b) in the DEX/CRH test, areas-under-the-curve (AUCs) and CORT delta (peak minus baseline) were significantly larger in RLA rats; and (c) increased sensitivity of RLA rats to CRH was associated with an increase in pituitary CRH receptors. Conclusions: Based on this model, and previously published clinical data, we suggest that hypersensitivity to $\mathrm{CRH}$, possibly linked to increased CRH receptor expression in the pituitary, could be a trait marker (or endophenotype) for depression, whereas nonsuppression in the DST could be a state marker (only present during depressive episodes).

\section{V5 \\ Effects of Mineralo-Corticoid Receptor Modulation by Spironolacton and Fludrocortisone on Cortisol Secretion and Learning Performance under a Stressful Learning Task}

\author{
U. Hemmeter, M. Giesler, B. Kundermann, A. Thum,
} B. Flux, P. Klee, A. Haag, J.C. Krieg

University of Marburg, Clinic of Psychiatry and

Psychotherapy, Marburg, Germany

Introduction: The activity of hypothalamus-hypophysis-adrenal (HPA) axis is regulated by mineralo- (MR) and glucocorticoid receptors. Furthermore, animal studies suggest that the glucocorticoid- and the MR-system are involved in learning and memory processes. Therefore, we conducted a study to evaluate the effects of a modulation of the MR-System on basal and stress induced cortisol secretion and on learning and memory function. Methods: In an intraindividual placebo controlled completely balanced repeated measurement design 24 healthy male subjects (range 20-30 years of age) received a single oral dosage of either $200 \mathrm{mg}$ spironolacton, $0.1 \mathrm{mg}$ fludrocortisone or placebo three hours before a stressful learning task. Number of trials, correct and false answers during the learning task and the number of correctly recalled associations at the end of the experiment were assessed. Cortisol samples were obtained from saliva before and until $120 \mathrm{~min}$. after the beginning of the stress task. Results: The application of $200 \mathrm{mg}$ spironolacton significantly increased baseline cortisol secretion compared to placebo and fludrocortisone. After the application of spironolactone cortisol secretion under stress was prolonged compared to fludrocortisone. In addition, there was a trend for a better learning performance under fludrocortisone. No differences between conditions for memory retrieval could be observed. Conclusion: Spironolacton is able to increase the basal activity of the HPA-axis and may prolong stress induced cortisol secretion. The observation of a faster learning performance under fludrocortison compared to spironolacton suggests that the modulation of the MR-function is related to learning performance probably mediated by cortisol.

\section{V6 \\ Romantic Love, Hypomania, and Sleep Pattern in Adolescents}

S. Brand, M. Luethi, A. von Planta, M. Hatzinger, E. Holsboer-Trachsler

Psychiatric University Clinics Basel, Depression Research Unit, Basel, Switzerland

Purpose: In adolescence, falling in love is an important prerequisite for acquiring psychosocial and psychosexual skills. Early-stage intense romantic love involves physiological, cognitive, emotional and behavioral dimensions: increased arousal, persistent thoughts for the beloved person, feelings of overwhelming joy, and efforts to sustain proximity to the person loved are prominent features of adolescents 
in a state of intense romantic love. The stage is comparable to a hypomanic-like state. However, no research has addressed this issue. Furthermore, no data are available with respect to sleep and sleeprelated dimensions such as mood. Method: 107 adolescents (mean age: $17.98 \pm 1.33$ ) took part in the study; 60 of them indicated they had recently fallen in love and experiencing intense romantic love, 47 had a longer-lasting relationship or were singles. Following a screening interview for psychiatric disorders, participants completed questionnaires related to hypomania and sleep habits. Additionally, they completed a sleep log for seven consecutive nights. Results: Compared to controls, adolescents in early-stage intense romantic love had increased scores on a hypomania scale, and recorded increased positive mood states in the mornings and in the evenings. These adolescents also recorded fewer hours of sleep with increased subjective sleep quality, lowered daytime sleepiness, and heightened concentration during the day. Conclusions: In adolescence, earlystage intense romantic love is comparable to a hypomanic state. This state is also reflected in altered sleep patterns, mood and daytime functioning. Consequently, in order not to bias data in research with adolescents, current stage of love should be taken into account.

\section{V7 \\ The Effects of Age upon Human Molecular Circadian Rhythms}

L. Pagani ${ }^{1}$, L. Waldmeier ${ }^{1}$, F. Meier ${ }^{1}$, J. Izakovic ${ }^{2}$, C. Cajochen ${ }^{3}$, A. Wirz-Justice ${ }^{3}$, S.A. Brown ${ }^{4}$, A. Eckert ${ }^{1}$

${ }^{1}$ Neurobiology Laboratory, Psychiatric University Clinic, ${ }^{2}$ Allergy Unit, Department of Dermatology, University Hospital, ${ }^{3}$ Center of Chronobiology, Psychiatric University Clinic, Basel, ${ }^{4}$ Institute of Pharmacology and Toxicology, University of Zurich, Switzerland

Purpose: Circadian oscillations in the expression of mammalian 'clock genes' are found not only in the suprachiasmic nucleus ( $\mathrm{SCN})$, the central circadian pacemaker, but also in peripheral tissues, e.g. skin fibroblasts. All of these cells possess independent cell-autonomous clocks of similar molecular makeup. The fact that fibroblasts use the same molecular components of the master clock could render them quite useful in uncovering circadian differences of genetic origin among individuals and populations. For human beings in general, it has been shown that circadian phase changes with age: individuals show increasingly early chronotypes as adulthood progresses. Nevertheless, the origin of this phenomenon is unknown. In our study, we addressed the question of whether fibroblasts reflect agerelated differences in circadian period length in vitro, that parallel changes in subject circadian phase in vivo. Methods: In an ongoing study, we are currently investigating period length of dermal fibroblasts taken from young and old subjects (young: younger than 30 years old; old: older than 60 years old; sex-matched). We have excluded extreme chronotypes by means of the Munich Chronotype questionnaire in both groups, because other factors in these subjects may influence period length per se. Two two-millimeter skin biopsy punches were collected from the buttock of the subjects. Skin fibroblasts were isolated and amplified from the biopsies, then infected with an engineered lentiviral circadian reporter (mBmal-1::luc) that permits the characterization of circadian rhythms in vitro by bioluminescence imaging. Measurements ( 3 per biopsy; $\mathrm{n}=6$ total) were conducted over a time period of 5 days.

Summary and Conclusions: Here we show the preliminary results of a small group of old and young subjects. Our findings will provide the first evidence whether age-related differences in circadian behavioural phase might be caused by underlying changes in the period of the circadian oscillator (e.g. shorter circadian period with age). The study also validates a novel method that has potential not only for diagnosis of circadian disorders, but also for assessment of future pharmacological interventions that modify or synchronize circadian clocks and thus could ameliorate sleep disorders. Support: Research supported by EU-CLOCK Grant from E.U. \#LSHM-CT2006-018741 and a grant from Désireé \& Niels Yde Fundation.

\section{V8}

\section{A Triple Transgenic Mice Model of Alzheimer's Disease with Plaques and Tangles}

V. Rhein'1, G. Baysang1, F. Meier1, L. Ozmen², E. Savaskan',

${ }^{1}$ Neurobiology Research Laboratory, Psychiatric University Clinic, ${ }^{2}$ Hoffmann-La-Roche AG, Pharma Research, Neurosciences, Basel, Switzerland; ${ }^{3}$ Alzheimer's and Parkinson's Disease Laboratory, Brain and Mind Research Institute, University of Sydney, Sydney, Australia

Alzheimer's disease (AD) is the most frequent form of dementia among the elderly and is characterized by neuropathological hallmarks of extracellular amyloid plaques and intracellular neurofibrillary tangles in the brain of $\mathrm{AD}$ patients. Amyloid plaques are composed of the amyloid-beta $(\mathrm{A} \beta)$ protein, derived from its precursor protein APP. Neurofibrillary lesions are formed from paired helical filaments composed of hyperphosphorylated tau protein, a microtubule-associated-protein. Importantly, current data indicate a complex relation between the amyloid pathology and pathology involving microtubule-associated protein tau during disease. Based on our previous findings, we hypothesize a direct impact of abnormally phosphorylated tau and $A \beta$ on proteins/enzymes involved in energy metabolism and mitochondrial respiratory chain. For this approach we are currently investigating the brains of double (APP (KM670/671NL)/PS2 (N1411)) and triple (APP (KM670/671NL)/ PS2 (N1411)/Tau (P301L)) transgenic mice. Mitochondrial respiration is studied by the measurement of the oxygen consumption with substrates and inhibitors specific for the mitochondrial chain complexes I and IV. In addition, enzyme activities of these complexes will be determined. Our preliminary findings indicate that at the level of mitochondria, the two defining neuropathological AD proteins, tau and $A \beta$, seem to act in a synergistic or additive way finally leading to/accelerating neurodegenerative mechanisms and cell death. Support: SNF grant 310000-108223 and Eli Lilly International Foundation grant to AE. 
V9

\section{Subgrouping Alcoholics According to their Age of Alcoholism Onset}

G.A. Wiesbeck, K.M. Dürsteler-MacFarland, S. Müller, S. Petitjean, F.M. Wurst

Department of Psychiatry, University of Basel, Switzerland

There is evidence that subgrouping alcoholics according to their age of alcoholism onset is clinically important. In order to elucidate the impact of this parameter we used four different approaches to study individuals with an ICD-10 diagnosis of alcohol dependence. We defined onset of alcoholism as the age when a minimum of three ICD-10 dependence criteria clustered for the first time. According to international practice an onset before 25 years of age was considered as early. In a first study $(n=76)$ we investigated the clinical applicability of Cloninger's biological concept of type 1/type 2 alcoholism in which age of onset is regarded as an crucial grouping variable. Subgrouping alcoholics according to their age of onset was not reflected by significant differences in type 1 and type 2 scores. In a second study $(\mathrm{n}=156)$ we investigated the relation between age of onset and treatment outcome. While there was a significantly higher rate of paternal alcoholism in individuals with early onset, we could not confirm previous reports of an association between age of onset and treatment outcome, as reflected by abstinence/relapse rates within one year after inpatient detoxification. In a third study $(\mathrm{n}=114)$ we investigated the genotypic frequencies of the $-141 \mathrm{C}$ Ins/Del polymorphism of the DAD2 receptor gene as well as the Bal I polymorphism of the DAD3 receptor. None of these gene variants was associated with age of alcoholism onset. Finally, in a fourth study we applied a three-axes approach to subgroup 51 alcoholics. We rated them on a clinical axis (early/late onset of alcoholism), on a personality axis (high/low sensation seeking), and on a biological axis (high/low dopamine receptor sensitivity). We identified a subgroup which was characterized by early alcoholism onset, high sensation seeking and high dopamine receptor sensitivity. Our data do not support the relevance of subgrouping alcoholics according to their age of alcoholism onset. We assume that positive reports in the literature are confounded by not controlling for important comorbidities. 


\section{Poster Presentations}

P1

\section{Quantitative Electrophysiological (qEEG) Differences in Subgroups from a Group or Individuals Rated 'At Risk' for a Psychotic Episode}

\author{
D. Bars ${ }^{1}$, U. Gschwandtner ${ }^{2}$, A. Riecher-Rössler', \\ V. Semenin' ${ }^{2}$, T. Abraham ${ }^{3}$, P. Fuhr ${ }^{4}$ \\ ${ }^{1}$ Private Practice, USA; ${ }^{2}$ Psychiatric Outpatient \\ Department, University Hospital, Basel, Switzerland; \\ Institute for Social and Behavioral Research, USA; \\ ${ }^{4}$ Department of Neurology, University Hospital, Basel, \\ Switzerland
}

Purpose of the Study: Longitudinal, post-hoc analysis of qEEG was conducted in a multiyear, multidisciplinary study (Basle FEPSY) investigating scientific bases for early identification of psychosis. In a literature review of neurophysiological studies and review articles, group differences in first episode and schizophrenic populations were evident; but few, if any, studies present pre-psychosis data. This study is unique because it evaluates qEEG data recorded prior to psychosis. The objective was to evaluate qEEG data as a predictor for development of psychosis in individuals assessed by clinical psychiatric examination to be at risk for schizophrenia. Methods Used: Four groups were previously using results from: a local screening instrument and the Brief psychiatric Rating Scale (BPRS). Groups consisted of: first episode patients; individuals at-risk for psychosis, and two control groups; a 'normal' group and individuals with other psychiatric disorders. Three main effects were evaluated: group, electrode site, and frequency band. The three way interaction; site band ${ }^{*}$ group, two way interactions, site ${ }^{*}$ group and site ${ }^{*}$ band and then the main effects were analyzed. Fast Fournier Transformed qEEG amplitude data were evaluated. Results: Three-way interactions were not significant for any of the bands. Several significant band"group interactions occurred, in lower $\alpha(p=0.032), \gamma 1$ $(p=0.009)$ and $\gamma 2(p=0.011)$ bands. Conclusions: This data suggest that increased resting neurophysiological activity may precede the first episodes of psychosis. It appears that qEEG analysis in conjunction with other neurophysiological imaging and clinical data may aid in the correct identification of individuals in very early stages of schizophrenia adding to the ability for earlier intervention in an extremely debilitating disorder.

This work was first presented at the XXVIIth International Congress of Clinical Neurophysiology, Edinburgh, UK, 10-14 September 2006.
P2

The Neuropsychological Assessment of the Risk for Psychosis in the Basel FEPSY Study

M. Pflueger, U. Gschwandtner, J. Aston, S. Borgwardt, R.-D. Stieglitz, A. Riecher-Rössler

Psychiatric Outpatient Department, University Hospital, Basel, Switzerland

Purpose of the Study: To investigate the cognitive capacity of individuals at risk for schizophrenia (IR) compared to healthy controls (HC) and to determine a subset of cognitive functions which discriminates best between the two groups. Methods Used: $\mathrm{N}=60 \mathrm{IR}$ and $\mathrm{N}=51 \mathrm{HC}$ were assessed with a neuropsychological test battery. Five tests were administered to assess attentional (CPT, TAP- Working Memory, TAP- Go/NoGo) and executive functions (WCST, ToH). In a first step all neuropsychological measures were adjusted for potential confounding factors (e.g. education, medication, and use of cannabis). Groups were compared by means of (M)ANOVA. A LOGIT regression procedure with backward stepwise elimination was then carried out in order to detect the subset of measures which best predicts the assignment to the at risk groups. Results: A MANOVA resulted in a general difference of the neuropsychological performance between IR and HC. Moreover, multiple statistically significant differences were found with post-hoc single measure analyses. HC always performed better than IR. By means of LOGIT regression, a number of variables were identified as the best predictors of the at risk status. Conclusion: Both executive and attentional functions are impaired in IR. The best prediction of 'at risk' status is achieved by assessing specific aspects of attention and executive functions.

This work was first presented at the 14th AEP Congress, Nice, 4-8 March 2006.

P3

Prolactin Serum Levels in Individuals at Risk for Psychosis, First Episode Patients and Depressive Controls
E. Rechsteiner, J. Aston, N. Bull, A. Riecher-Rössler
Psychiatric Outpatient Department, University Hospital, Basel, Switzerland

Purpose of the Study: While it is well documented that hyperprolactinemia is associated with neuroleptic treatment in schizophrenia, prolactin levels in drug-naive patients with first episode of psychosis are less well investigated and to our knowledge have never been investigated in individuals at risk for psychosis. The objective was to compare prolactin serum levels in individuals at risk of psychosis, patients with a first episode of psychosis and depressive controls. Methods Used: Prolactin serum levels were measured in the framework of the Basel FePsy project (FrühErkennung von PSYchosen) in 27 patients with first episode psychosis, 43 individuals at risk of psychosis and 7 depressive controls. All types of medication prescribed before measurement of blood levels were documented. Results: 14 (51.8\%) out of 27 first episode patients showed increased prolactin serum levels, $6(22.2 \%)$ of them were neuroleptic-naive, $6(22.2 \%)$ had been taken low doses of 
olanzapine or risperidon sporadically or for less than 3 months, one had olanzapine for 5 months, another risperidone for 2 years. $11(25.6 \%)$ out of 43 patients with prodromal symptoms showed increased prolactin serum levels, $10(23.3 \%)$ of them were neuroleptic-naïve, one $(2.3 \%)$ had neuroleptics. All depressive controls showed normal prolactin levels. Conclusion: In both groups, individuals at risk and first episode patients, about one fourth had increased prolactin serum levels although they had never been treated with any medication causing hyperprolactinemia, except 3 of them who had low dose Chlorprothixen for a max. of 2 days. If these findings could be confirmed, they would indicate that hyperprolactinemia is not necessarily due to neuroleptics or frank psychotic breakdown, but could be a preexisting condition. The reason for this could be general mental stress or a change in the HPAAxis activity associated with the disease process itself.

This work was first presented at the DGPPN Congress, Berlin, 22-25 November 2006.

\section{P4}

First Signs and Symptoms in Individuals at Risk for Psychosis and Patients with a First Episode of Psychosis

\section{J. Aston, N. Bull, U. Gschwandtner, M. Pflüger, M. Drewe, R.-D. Stieglitz, A. Riecher-Rössler \\ Psychiatric Outpatient Department, University Hospital, Basel, Switzerland}

Purpose of the Study: The aim of the FePsy study is to improve the risk assessment and prediction of psychosis. Individuals referred to our specialised clinic suspected to be in a prodromal stage of schizophrenia or to be psychotic are screened and if included, a comprehensive multimodality (early recognition interview, psychopathology, neuroradiology, neurophysiology, neuropsychology) investigation is done. Then the individuals at risk (IR) are followed up for at least 5 years. Here we report findings from the early recognition interview on first self-perceived signs and symptoms and on duration of the (putative) prodrome. Methods Used: We developed an interview for the early recognition of psychosis to assess early signs and symptoms, as well as known risk factors for schizophrenia such as genetic risk. One section of this interview explores when the subject first noticed any change in well-being and what the first signs and symptoms were. We report on these findings from 62 IR, 37 patients with a first episode of psychosis (FE) and 16 controls with depression (DC). Results: The median of the time from first self-perceived symptoms up to the interview was 48 months for the IR and 36 months for the FE, the mean values were 61 (SD 63.9) for the IR and 70 (SD 86.5) for the FE. The most frequently mentioned first symptoms in the IR were 'loss of drive and energy', 'difficulties concentrating' and 'social isolation'. In the FE they were 'feeling depressed', 'becoming more nervous', 'more irritable' and 'social isolation'. Conclusion: The results are in accord with other literature on the duration of the prodrome such as the retrospective Mannheim ABC Study which reported a duration of 3.5 years, and comparable first signs and symptoms. In a further step, the specificity of these symptoms will be evaluated by comparing IR and FE with a control group of patients with depression.

This work was first presented at the 5th International Conference on Early Psychosis, Birmigham, UK, October 4-6, 2006.

\section{P5}

\section{Interviewing Relatives as a Contribution to the Risk Assessment for Psychosis in the Basel FEPSY Study}

\author{
J. Aston, N. Büchel, U. Gschwandtner, M. Pflüger, \\ M. Drewe, A. Riecher-Rössler
}

Psychiatric Outpatient Department, University Hospital, Basel, Switzerland

Purpose of the Study: The aim of the study is to improve risk assessment for psychosis. At risk individuals (IR) are identified in a screening procedure, and if included, a comprehensive multilevel investigation is done including the extensive, newly developed 'interview for the early detection of psychosis' (IFP) covering case history, risk factors, early signs and prodromal symptoms. In order to assess childhood abnormalities more accurately, we also developed an interview for close relatives. Here we report first preliminary results. Methods Used: The interview for relatives covers deviations from normal development in infancy and preschool age, psychosocial factors, behavioral and learning difficulties at primary school age. Agreement between answers covering identical topics given by the IRs in the IFP and the relatives was determined using the kappa coefficient. Summary of the Results: Out of the 58 IR included from the 1.3.2000-28.2.2003, 15 relatives (mainly mothers) could be interviewed. Acceptance by relatives interviewed and feasibility were good. However, many IR did not give permission for their relatives to be interviewed, reasons were for example 'do not want parents to know they have been in contact with a psychiatric institution' or 'conflicts with parents'. With the relatives' interview, additional information was obtained mainly regarding milestones in infancy and problems at school. Conclusion: The interview is a useful tool for assessing developmental abnormalities in early childhood and school age, and contributes to the overall assessment of risk for psychosis. The difficulties encountered in obtaining permission from the IR to interview their relatives might reflect fear of psychiatric institution and of stigmatisation.

This work was first presented at the XIIIth Biennial Winter Workshop on Schizophrenia Research, 4-10 February 2006, Davos, Switzerland.

\section{P6}

\section{Specific Patterns of Gray Matter Atrophy and Elevated Cerebral Blood Flow in Left Sided Language Regions in Formal Thought Disorder}

\author{
H. Horn ${ }^{1}$, A. Federspiel', M. Wirth', T. Müller ${ }^{1}$, \\ R. Wiest ${ }^{2}$, W. Strik ${ }^{1}$
}

${ }^{1}$ University Hospital of Psychiatry, ${ }^{2}$ Institute of Diagnostic and Interventional Neuroradiology, University of Bern, Bern, Switzerland

Purpose of the Study: Formal thought disorder is a central feature of schizophrenia, but its neural foundation still remains unclear. The understanding of the neural foundation of this important symptom is essential to find specific treatments without unnecessary side effects. 
Methods: Thirteen right-handed schizophrenic patients with different severity of formal thought disorder and thirteen matched healthy controls were investigated by voxel based morphometry and MRI perfusion measurement with Arterial Spin Labeling (ASL). Results: We found hyperperfusion to be linked to the severity of formal thought disorder in the left sided language system involving frontal, temporal and parietal areas. Gray matter atrophy was linked with the severity of formal thought disorder bilateral in the superior temporal sulcus, the angular gyrus, the anterior cingulate gyrus and the inferior temporal gyrus. For the left hemisphere the posterior superior temporal gyrus and the supramarginal gyrus and for the right hemisphere the precuneus showed gray matter reduction with increasing formal thought disorder. The results for perfusion and volume mainly involve frontal, brain areas strongly connected to language. Conclusion: In formal thought disorder the specific gray matter atrophy in temporo-parietal regions may be understood as a risk factor for decompensation of the left sided language system leading to a transient hyperperfusion of the Broca and Wernicke area as a sign of state related dysfunction.

\section{P7}

\section{The Early Context Effect - A Novel Parameter to Examine Semantic Dysfunctions in Schizophrenia}

\author{
M. Wirth ${ }^{1}$, H. Horn 1 , T. Koenig ${ }^{1}$, A. Razafimandimby ${ }^{3}$, \\ M. Stein ${ }^{1}$, T. Müller ${ }^{1}$, A. Federspiel', B. Meier ${ }^{2}$, T. Dierks ${ }^{1}$, \\ W. Strik \\ ${ }^{1}$ University Hospital of Psychiatry, ${ }^{2}$ University of Bern, \\ Institute of Psychology, Bern, Switzerland; ${ }^{3}$ Group \\ d'Imagerie Neurofonctionnelle, UMR 6194, CNRS, CEA, \\ Universities of Caen and Paris 5, Caen, France
}

Loose semantic associations are considered a core deficit in schizophrenia however the neurophysiologic correlates remain unclear. Some explanations have proposed that wider or faster activation spread in semantic networks might account for semantic disturbances in particular in patients with though disorder. Previous Event-related potential (ERP) studies have implemented semantic priming paradigms to elucidate the dysfunctional semantic processing and showed normal, increased or decreased semantic priming in schizophrenic patients in the N400 priming effect known to reflect controlled (attention-based) semantic retrieval. Because only a few ERP studies suggest early priming effects, there was no electrophysiological measure of the initial and potentially more automatic access to semantic information. We implemented a semantic priming paradigm to examine the existence of an early context effect and its spatiotemporal characteristics. For stimulation we applied a passive reading task consisting of German noun-noun word pairs. The word pairs varied in their degree of semantic relation; related (housebuilding) vs. non related (building-shoe). Our results demonstrate an early context effect in the P1-N1 time period, about $120-150 \mathrm{~ms}$ post visual word onset. Moreover we could clarify that the effect is semantic in nature and emerges from automatic retrieval in a temporal-prefrontal semantic network. The early context effect therefore provides a promising neurophysiologic correlate in the examination of dysfunctional semantic retrieval in psychiatric as well as neurological patients.
P8

A Glutathione Precursor, N-Acetyl-Cysteine, Modulates Mismatch Negativity Generation in Schizophrenia Patients

\author{
S. Lavoie 1,4, M.M. Murray 2,3 , M. Knyazeva ${ }^{3}$, P. Bovet, \\ T. Buclin ${ }^{5}$, P. Conus ${ }^{4}$, P. Deppen ${ }^{1,4}$, R. Meuli, A. Solida ${ }^{1,4}$, \\ M. Cuenod ${ }^{1,4}$, K.O. Do $0^{1,4}$ \\ ${ }^{1}$ Center for Psychiatric Neuroscience, ${ }^{2}$ Functional \\ Electrical Neuroimaging Laboratory, ${ }^{3}$ Radiology Service, \\ ${ }^{4}$ Department of Psychiatry, ${ }^{5}$ Department of Clinical \\ Pharmacology, Lausanne, Switzerland
}

Schizophrenia patients exhibit deficits in low-level processing, including pitch discrimination. This deficiency manifests in auditory evoked potentials (AEPs) as an impaired mismatch negativity (MMN), an electrophysiological response to infrequent target stimuli interspersed among frequent standard stimuli that typically peaks about $100 \mathrm{~ms}$ post-stimulus onset. NMDA receptor antagonists have been shown to block MMN generation in both animals and humans, and NMDA dysfunction has been linked to the underlying pathophysiology of schizophrenia. A parallel line of evidence indicates that glutathione (GSH) regulation is perturbed in schizophrenia patients at the gene, protein and functional levels [1]. This GSH dysregulation leads to NMDA receptors' hypofunction through interaction with their redox site [2]. The present study aimed to modulate GSH levels in schizophrenia patients and assessed the effects of such a modulation on MMN generation mechanisms. N-acetyl-cysteine (NAC), a GSH precursor, was administered to schizophrenia patients, using a double-blind cross-over protocol. One group received NAC ( $2 \mathrm{~g} /$ day) for 60 days and then placebo for another 60 days, and vice-versa for the second group. AEPs from patients were recorded at the onset of the protocol, at the point of cross-over, and at the end of the study. Participants were instructed to manually respond to target stimuli ( $2 \mathrm{kHz}$ pure tones occurring $20 \%$ of the time among $1 \mathrm{kHz}$ pure tones). Analyses of AEPs recorded at protocol onset indicated that patients $(n=11)$ were significantly impaired in generating the MMN relative to age-matched controls $(n=11)$. Specifically, the global field power (GFP), an index of AEP magnitude, was measured over the $70-155 \mathrm{~ms}$ post-stimulus interval and submitted to an analysis of variance (ANOVA). There was a significant interaction between population and stimulus frequency, indicating impaired MMN generation in patients at protocol onset. Analyses of AEPs recorded during administration of NAC $(n=7)$ versus placebo $(n=7)$ revealed the efficacy of this GSH precursor in modulating MMN generation mechanisms. ANOVA of GFP over the $70-155 \mathrm{~ms}$ post-stimulus interval, using stimulus frequency and treatment as within-participants variables, revealed a significant interaction and indicated that NAC can ameliorate MMN generation. We discuss these results in terms of potential therapeutic strategies for schizophrenia.

\section{References}

1. Tosic M et al: Schizophrenia and oxidative stress: glutamate cysteine ligase modifier as a susceptibility gene. Am J Hum Genet 2006;79:586-592.

2. Steullet $\mathbf{P}$ et al: Synaptic plasticity impairment and hypofunction of NMDA receptors induced by glutathione deficit: relevance to schizophrenia. Neuroscience 2006;137:807-819. 
P9

\section{Prosody Identification and Processing in Paranoid Schizophrenia}

\section{D.R. Bach}

Department of Psychiatric Neurophysiology, University Hospital of Psychiatry, Bern, Switzerland

Identification of emotions in affective prosody is reduced in paranoid schizophrenia. Little is known about the neural underpinnings of this deficit. Since emotion identification tasks that are used in schizophrenia research require naming and therefore explicit processing of stimuli, which is different from real-life situations, we were interested in comparing implicit and explicit processing of affective prosody. (1) In a first event-related fMRI study, we addressed implicit and explicit processing of anger and fear prosody in 16 healthy subjects. We found emotion-specific activation in the implicit condition, and additional activity related to attention and response in the explicit condition. (2) In a behavioural experiment on paranoid schizophrenia, identification of affective prosody and facial affect was examined. Specific deficits and category confusions were found for schizophrenic subjects, compared to healthy control or depressive subjects. (3) These results have implications for an ongoing fMRI study on prosody processing in paranoid schizophrenia.

\section{P10}

\section{Neuronal Model of Hierarchical Concept Formation and Retrieval in Healthy Controls and Patients with Thought Disorder}

\author{
T. Koenig ${ }^{1}$, W. Senn ${ }^{2}$, W. Strik ${ }^{1}$ \\ ${ }^{1}$ University Hospital of Psychiatry, ${ }^{2}$ Physiological Institute, \\ University of Bern, Bern, Switzerland
}

Purpose of the Study: The formation of hierarchically related concepts may be fundamentally disturbed in schizophrenic patients with thought disorders. These patients often have difficulties associating different objects to an abstract superordinate category. They may either have difficulties to distinguish items that belong to a common superordinate concept, or, contrary, they may have difficulties associating similar items belonging to the same superordinate concept. A biologically plausible theoretical model of the formation and retrieval of categorical information may help to understand and classify the clinical observations and rephrase them in terms that are tangible by the methods of biological psychiatry. Methods: We developed a neural network model that can produce a certain level of abstraction based on Hebbian learning of input stimuli. Depending on the demand, the network responds to a stimulus by either representing the more abstract supercategory of the stimulus, only the stimulus category itself, or all subordinate individual items belonging to the same stimulus category. Results: Simulations showed that hierarchically organized attractor networks may spontaneously form out in response to a statistically structured set of input patterns. Wide neuronal attractors represent individual stimulus categories, while the intersection of these attractors represents the common supercategory. The level of abstraction can be controlled by tuning global inhibition: weak inhibition stabilizes the wide, low level attractors; strong inhibition stabilize the narrow, high level attractors. Conclusions: Hierarchical attractor networks offer a new framework to study concept formation and retrieval in healthy subjects and patients with thought disorder.

\section{P11 \\ Global Dimensionality of Multichannel EEG in Schizophrenic Patients is Reduced by Neuroleptic Medication}

\author{
T. Waltinger ${ }^{1,2}$, D. Lehmann'2, P.L. Faber' ${ }^{2}$, T. Koenig ${ }^{3}$, \\ K. Kochi ${ }^{2}$, and W. Strik ${ }^{3}$ \\ ${ }^{1}$ Psychiatriezentrum Münsingen, Bern, ${ }^{2}$ The Key Institute \\ for Brain-Mind Research, University Hospital of Psychiatry \\ Zurich, ${ }^{3}$ University Hospital of Psychiatry, Bern, Switzerland
}

Purpose: The original concept of schizophrenia as a functional dissociation (Bleuler's 'loosening of associations') is in agreement with reports of increased EEG dimensionality in these patients, indicating a lessening of cooperativity of brain processes: we found increased dimensionality and Omega-complexity in EEG of acute, productive, medication-naïve schizophrenics compared to controls. Recently, decreased dimensionality was reported in partly medicated, chronic schizophrenics. The present study on such patients intended to clarify the effect of neuroleptic medication on EEG dimensionality. Methods: The EEG files at the Bern University Hospital of Psychiatry between 2000 and 2006 were searched for schizophrenia diagnoses (ICD F 20) while excluding affective symptomatology, other co-morbidity, and drug addiction. Eventually, 23 cases with sufficient artifact-free EEG data were available (mean age 37.9, $\mathrm{SD}=10.0,12$ women) who received neuroleptic medication during the last 10 days before EEG recording (on average/patient on 8.6 days) and who received no other types of medication during the last 3 days before EEG recording. On average/patient, 196 seconds of artifact-free 19-channel EEG was available for analysis with Global Dimensional Complexity (GDC) and Omega-complexity. Neuroleptic medication was recomputed into chlorpromazine equivalents (average during last 3 days: $357 \mathrm{mg} /$ day/patient). Spearman rank correlations were computed between GDC and Omega versus equivalent levels. Results: The magnitude of the chlorpromazine equivalent of the neuroleptic medication during the last 3 days correlated negatively (Spearman $\mathrm{R}=-0.475, \mathrm{p}=0.02$ ) with GDC, but not significantly with Omega-complexity. There were no significant correlations between GDC with gender, age, handedness, and duration of current episode. Conclusions: With increasing level of neuroleptic medication, EEG global dimensionality in the patients systematically decreased, thus, in the direction of normalization. Our results suggest that recently reported decreased dimensionality in chronic schizophrenia might be the effect of neuroleptic medication. 


\section{P12}

\section{Increasing Second Language Proficiency Reduces Activation Differences between First and Second Language}

\author{
M. Stein 1, A. Federspiel', C. Lehmann'1, T. Koenig1, \\ R. Wiest ${ }^{2}$, W. Strik' 1 D. Brandeis ${ }^{3,4}$, T. Dierks ${ }^{1}$ \\ ${ }^{1}$ University Hospital of Psychiatry, Department of \\ Psychiatric Neurophysiology, ${ }^{2}$ Institute of Diagnostic and \\ Interventional Neuroradiology, Inselspital, University of \\ Bern, Bern, ${ }^{3}$ Brain Mapping Research, Department of \\ Child and Adolescent Psychiatry, University of Zürich, \\ ${ }^{4}$ Center for Integrative Human Physiology, University of \\ Zürich, Zürich, Switzerland
}

Differences in representation and processing of a bilingual's first (L1) and second (L2) language have been investigated in a number of studies. One of the potential factors influencing the degree of separation and overlap between L1 and L2 is second language proficiency. In the present longitudinal event-related functional magnetic resonance imaging (fMRI) study, we addressed the question whether activation differences between single word processing in L1 and L2 are minimized with increasing second language proficiency. Native English speaking exchange students learning German in Switzerland were examined once at the beginning of their stay and a second time about 5 month later, when their second language skills had significantly increased. With low second language proficiency, we found higher activation for $\mathrm{L} 2$ in a distinct cortical network located in bilateral inferior frontal gyri, left middle frontal gyrus, inferior parietal lobule, SMA and cingulated gyrus. When second language proficiency was higher, the activation of L2 was significantly reduced as compared to day 1 in most of these regions. Our results demonstrate plasticity in the adult language system and support the view that second language proficiency influences the degree of separation and overlap of single word processing in different languages.

\section{P13}

\section{Event Related fMRI of Primary- and Higher Cognitive Cerebral Functions with Arterial Spin Labeling (ALS) Perfusion}

\author{
A. Federspie/1, T. Dierks' ${ }^{1}$, C. Kiefer ${ }^{1}$, H. Rao ${ }^{2}$, J.J. Wang ${ }^{2}$ \\ 1Department of Psychiatric Neurophysiology, University \\ Hospital of Psychiatry, Bern, Switzerland; ${ }^{2}$ University of \\ Pennsylvania, Philadelphia, Pa., USA
}

Introduction: In the field of functional neuroimaging the use of blood oxygen level-dependent (BOLD) fMRI has been extremely successful. There is evidence of a coupling between neuronal activation, metabolism, and blood flow which is still not fully understood. This could pose a limitation for this fMRI method especially in the clinical field where factors influencing the coupling may be altered. One possible way to overcome this shortcoming is the use of eventrelated cerebral blood flow (CBF) Perfusion fMRI experiments. Several studies demonstrated the feasibility of event-related Perfusion
fMRI experiments using Arterial Spin Labeling (ASL) [1-4]. In these studies one single trial type was used in separate runs to reconstruct the hemodynamic response function (HRF). In the present pilot study we investigated four different trial types within the same run. The following hypothesis was formulated: it is possible to localize the activation evoked by each trial type separately and to reconstruct the HRF of all trial types within the same run. If these hypotheses hold, then event related CBF fMRI offers several advantages as compared to block design experiments; i.e. intermixing of trials of different types and the option to categorize each trial according to the behaviour of the subjects. The later is of importance when comparing controls with patients group. Methods: Four subjects were measured with pulsed ASL (PASL) [5, 6]. The stimulus material, displayed through a MR-compatible LCD goggle system, consisted in three trial types presented in randomized order: (a) full-field $8 \mathrm{~Hz}$ checkerboard, (b) apparent radial moving dots at $4 \mathrm{~Hz}$ and (c) picture of human faces. All Stimuli lasted $3 \mathrm{sec}$ followed by $6 \mathrm{sec}$ fixation cross. A 'null event' was added to allow for a randomized inter stimulus interval. MR scanning was on a 3.0 T Siemens Trio system, using the 12-channely array head coil as receiver and body coil as transmitter. The whole study included a structural 3D MRI (3D Turbo-flash). A PASL sequence was a modified FAIR technique, as described previously [7]. A gradient-echo EPI sequence was used for image acquisition $(\mathrm{FOV}=23 \mathrm{~cm}, 64 \times 64$ matrix, TR $/ \mathrm{TE}=2500 / 11 \mathrm{~ms}, 12$ slices of $6 \mathrm{~mm}$ thickness with $1.5 \mathrm{~mm}$ gap, GRAPPA 2, 640 volumes, scan time $26.40 \mathrm{~min}$ ). PASL image series were first co-registered and smoothed with a $10 \mathrm{~mm}$ Gaussian-Kernel, and then pair-wise subtracted. CBF was quantified as described elsewhere [8]. All voxels with $p<0.01$ were considered as putative region of interest and used for the subsequent HRF reconstruction; for this a general linear model was used to estimate the finite impulse response (FIR) associated with each of the 3 trial type. Results and Discussion: The localization of brain activity evoked by the stimulus condition A, B and C was found to be at the expected cortical regions: i.e. early visual cortex for condition A (fig. 1), MT/V5 for condition B (fig. 2) and right fusiform gyrus for condition $\mathrm{C}$ (fig. 3). The reconstruction of the HRF for each stimulus condition shows an expected temporal pattern. These results demonstrate that it is possible to deconvolve the response function from a time series that contains the information of more than two stimuli. Furthermore, the results also show that it is possible to correctly localize the cerebral activation evoked by primary stimuli (i.e. checkerboard) as well as for higher cognitive stimuli (i.e. face processing). Event related fMRI with PASL comprising different stimulus modalities is feasible.

\section{References}

1. Liu HL, et al: Magn Reson Med 1999;42:1011-1013.

2. Liu HL, et al: Magn Reson Med 2000;43:768-772.

3. Yang Y, et al: Neuroimage 2000;12:287-297.

4. Liu TT, et al: Neuroimage 2002;16:269-282.

5. Detre JA, et al: Magn Reson Med 1992;23:37-45.

6. Wang J, et al: Magn Reson Med 2003;49:796-802.

7. Wang J, et al: J Magn Reson Imaging 2003;18:404-413.

8. Federspiel A, et al: J Neural Transm 2006;113:1403-1415. 

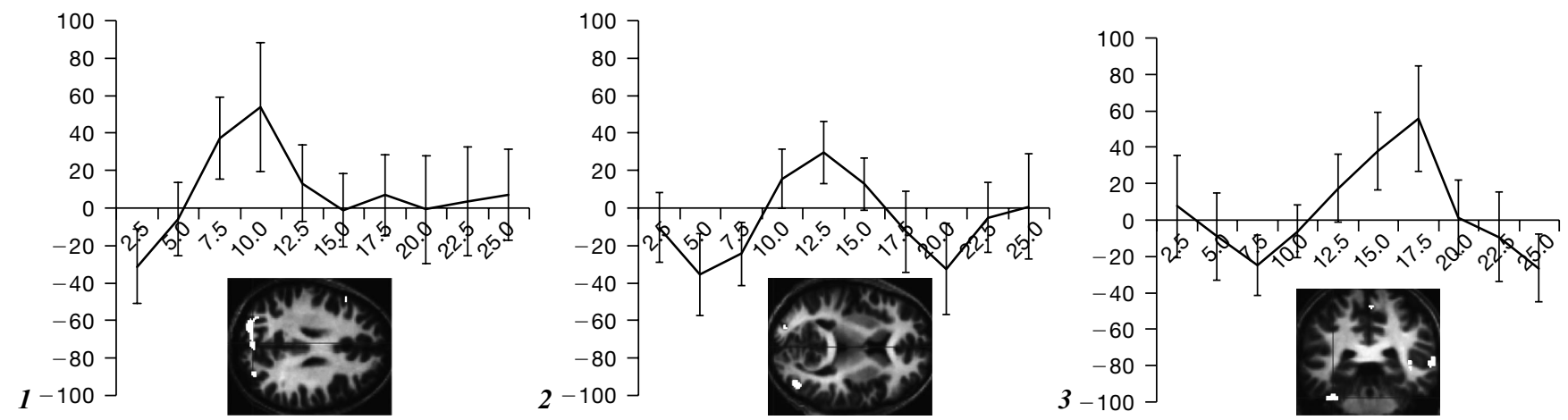

Fig. 1-3 for Abstract P13. White: activated area due to checkerboard-, motion and face processing paradigm with peristimulus time.

\section{P14 \\ Effect of Serotonin on GABAergic Interneuron Migration in the Embryonic Cortex}

\section{A. Dayer \\ Department of Adult Psychiatry, Geneva, Switzerland}

Increasing evidence has revealed that serotonin (5-HT) is not only a critical neurotransmitter in the regulation of mood and anxiety but is also a potent developmental signal. Recent studies in humans suggest that a shift toward pathological states, such as depression could occur when individuals carrying the short allele of the 5-HT transporter gene are exposed to significant environmental stressors. However the timing and the nature of the neurobiological abnormalities underlying an alteration of serotonin homeostasis remain to be elucidated. Recent data revealed that mice mutant for the 5-HT transporter gene display profound alterations in neocortical cell density in numerous cortical regions suggesting a perturbation in neuronal migration. We make the hypothesis that an excess of 5-HT during the late phases of cortical development could affect the migration and early dendritic development of cortical interneurons. To test this hypothesis we used the GAD65-GFP transgenic mice, which represent a valuable tool to study interneuron migration in vivo. At embryonic day E17.5 a large fraction of GAD65-GFP interneurons are observed in the subplate and displayed the typical morphology of interneurons radialy migrating towards the cortical plate. These GAD65-GFP interneurons were located in close contact to serotoninergic raphe fibers indicating that these cells must navigate through this serotonin-rich region before reaching the upper cortical plate. Time-lapse imaging in E17.5 cortical slices revealed that an excess of 5-HT dramatically impairs their migration. When 5-HT $(100 \mu \mathrm{M})$ was applied to the slice, the migration velocity of GAD65-GFP+ cells was dramatically reduced with a statistically significant reduction of more then $50 \%$ in the mean migration speed $(47.1 \mu \mathrm{M} / \mathrm{h}$ in controls compared to $22.1 \mu \mathrm{M} / \mathrm{h}$ after 5-HT application). Furthermore 5-HT application significantly reduced the mean neurite length of migrating cells suggesting that 5 -HT could induce a growth cone collapse and impair migration through this mechanism. These data open the possibility that an alteration in 5-HT homeostasis during development could induce neuro-developmental abnormalities.

\section{P15}

\section{Rest-Activity Cycles, Negative Symptoms and Cognitive Function in Schizophrenia - Preliminary Results}

\author{
V. Bromundt ${ }^{1}$, A. Kill ${ }^{2}$, G. Stoppe ${ }^{2}$, A. Wirz-Justice ${ }^{1}$ \\ ${ }^{1}$ Centre for Chronobiology, ${ }^{2}$ Department of General \\ Psychiatry, Psychiatric University Clinics, Basel, Switzerland
}

Negative symptoms in schizophrenia are characterised by reduced affect and emotion, lack of motivation, energy and ability to experience pleasures. These symptoms may also occur in people with sleep disturbances, which is also a common complain in schizophrenic patients. Thus, we are currently investigating the correlation between characteristics of the rest-activity cycles, negative symptoms and cognitive function in schizophrenic outpatients with minimal positive symptoms. The patients are keeping sleep diaries over a period of 21 days along with a recording of their rest-activity cycles by actigraphy. Saliva samples during 3 days (interspersed by 7-day interval) are collected to determine the onset of melatonin secretion as an objective marker for the phase of circadian rhythms. Moreover, clinical interviews and standardised questionnaires (BPRS, PANSS, SAS, PSQI) are conducted to assess clinical status, and medication and sociodemographic data are recorded. Cognitive functioning is being assessed by neuropsychological tests (Trail Making Test A + B, Stroop interference task, verbal fluency test). The rest-activity cycles in five patients treated with the atypical antipsychotic risperidone showed abnormalities, in particular frequent awakenings during the main sleep episode. The circadian phase, in 2 patients so far, showed a considerable delay. So far, we have found a weak relationship between the negative symptoms and the degree of the disturbances in rest-activity cycles as measured by the interdaily stability index in six patients. On the other side, we have observed a significant correlation between the interdaily stability index and executive cognitive functioning as measured by the Stoop interference task, while the correlation with the other neuropsychological tasks did not yield significance. From these preliminary results we conclude that disturbed-rest activity cycles may have a significant negative impact on cognitive functioning, particularly for executive cognitive performance, in schizophrenic patients. 


\section{P16}

\section{Lost in Time}

J. Späti, K. Blatter, M. Münch, V. Knoblauch, C. Cajochen

Centre for Chronobiology, Psychiatric University Clinics,

Basel, Switzerland

Introduction: Knowing of our position in time (i.e., temporal orientation) helps us adequately interact with the environment and may contribute to the daily entrainment of the circadian pacemaker to a near $24 \mathrm{~h}$ day. In this study, we investigated subjective clock-time estimates at different circadian phases during prolonged wakefulness in healthy young and older volunteers under constant routine conditions. Methods: Time-of-day estimates were collected in 16 young (8m, 8f; 20-31 years) and 16 older (8m, 8f; 57-74 years) healthy volunteers during $40 \mathrm{~h}$ of extended wakefulness under constant routine conditions in an environment devoid of temporal cues. At intervals of approximately $3.75 \mathrm{~h}$ subjects were asked to give a verbal estimate on time-of-day. Results: In general, an overestimation of clock time was found in both age groups, with significantly higher values for the older group (young: $0.4 \pm 0.3 \mathrm{~h}$ vs. older: $1.5 \pm 0.3 \mathrm{~h}, \mathrm{p}<0.05$ ). Estimation errors varied in adiurnal fashion in both age groups, the oscillation roughly paralleling the time course of core body temperature. However, a significant interaction between elapsed time awake $\mathrm{x}$ age group was found (rANOVA, $\mathrm{p}<0.05$ ). Post-hoc analyses revealed significantly higher magnitudes of estimation errors for the older at $8.6,12.4$, and $16.1 \mathrm{~h}$ of elapsed time of wakefulness $(\mathrm{p}<0.05)$; beyond $16.1 \mathrm{~h}$ elapsed time into protocol, the mean estimation error in the young group increased up to the level found in the older subjects group. Conclusion: Temporal orientation was more impaired in the older than in the young volunteers, particularly from the afternoon till late evening (16:30-24:00 h). However, time-of-day estimates in young people seem to be more susceptible to the effects of elevated sleep pressure than in the older.

\section{P17 \\ Rest-Activity Cycles and Circadian Rhythms in Women with Borderline Personality Disorder - Preliminary Results}

\section{S. Kyburz' ${ }^{1}$ V. Bromundt ${ }^{1}$, C. Cajochen ${ }^{1}$, G. Dammann ${ }^{2}$, A. Wirz-Justice ${ }^{1}$ \\ ${ }^{1}$ Centre for Chronobiology, Psychiatric University Clinics, Basel, ${ }^{2}$ Psychiatric Clinic, Münsterlingen, Switzerland}

Borderline Personality Disorder (BPD) is characterized by impulsive aggression, instability of mood and interpersonal relationship, unstable self-image, depression, anxiety, repeated self-injurious behaviour and chronic suicidal tendency. BPD patients often report to suffer from fatigue problems and sleep disturbances. Thus, we are currently investigating the effect of possible circadian rhythm disturbances on the above mentioned symptoms in BPD patients. Rest-activity cycles are being measured in outpatients diagnosed with BPD by DSM-IV criteria over a period of 21 days by actimetry along with daily questionnaires about their sleep quality, mood, sleepiness and tendency for self-injury. In addition, saliva samples during 3 days (each separated by a 7-day interval) are being collected to determine the onset of melatonin secretion as a marker for an objective estimation of the circadian phase position. In the course of the 21-day period clinical interviews and standardised questionnaires (BPI, PSQI) are conducted to assess clinical status, and the medication as well as sociodemographic data are recorded. Preliminary analyses of the actimetry and melatonin data have shown that many patients with BPD have abnormal rest-activity cycles and circadian rhythms. As an example we are presenting data of three BPD patients, ranging from very poor interdaily stability of rest-activity cycles and disturbances in sleep parameters to normal circadian patterns. Interestingly, the degree of these differences is correlated with the degree of the severity of BPD. Moreover, we found a strong and significant correlation between the depressive mood and the urge to intentional self-injury. We conclude that the observed alterations in the rest-activity cycles are related to circadian disturbances in BPD patients and that the degree of these 'circadian' symptoms are related to the degree of BPD symptoms. We think that light therapy will be a successful treatment to correct circadian rhythm disturbances as well as to diminish the urge for self-injury via its antidepressive effect in BPD patients.

\section{P18 \\ Effects of 40-hour Sleep Deprivation on Short-Term Memory in a Constant Routine Protocol}

D. Anders ${ }^{1}$, S. Vollenweider ${ }^{1}$, D.J.-F. De Quervain ${ }^{2}$, A. Wirz-Justice ${ }^{1}$, K. Kräuchi $^{1}$

${ }^{1}$ Centre for Chronobiology, Psychiatric University Clinics, Basel, '2Division of Psychiatric Research, University of Zürich, Zürich, Switzerland

Introduction: Circadian and homeostatic aspects of sleepiness regulation can influence the outcome of diverse neurocognitive performance tests. We investigated the influence of these components on short-term memory during 40-hour of sleep deprivation. Methods: Twelve healthy women (luteal phase; age: 20-31 year) completed a stringently controlled 40 -hour constant routine protocol. Core body temperature (CBT) was continuously measured, subjective sleepiness was rated on the Karolinska Sleepiness Scale at half-hourly intervals and performance in a forward digit span (F) and backward digit span (B) test was evaluated two-hourly. Results: Sleepiness ratings were highest during the CBT minimum and remained at a higher level on the 2nd day compared with the 1st day (sleep deprivation effect). Performance of $\mathrm{F}$ showed a circadian trough $6 \mathrm{~h}$ after the CBT minimum on day 2 (27.5 h after lights on) but not on day 1 ( $3.5 \mathrm{~h}$ after lights on). Performance of B showed a narrow circadian trough $2 \mathrm{~h}$ after the CBT minimum (23.5 h after lights on). This ruled out detection of a potential circadian trough $24 \mathrm{~h}$ earlier (sleep phase). No significant differences in $\mathrm{F}$ and $\mathrm{B}$ occurred between day 1 and 2 (no sleep deprivation effect). Conclusion: There is a circadian component in the performance of $\mathrm{F}$ that is visible only on the 2nd day when sleep pressure is high. Sleep deprivation-induced sleepiness alone seems to play a minor role in this test of short-term memory - only its interaction with the circadian trough worsens short-term memory. The circadian modulation of a less complex task (F) seems to be larger than that of a more complex one (B). Furthermore, the results do not 
support a direct effect of CBT on F and B performance. Support: Research supported by SNF Grant \#3100A0-102182 to K.K. and the Gottlieb Daimler and Karl Benz Foundation.

\section{P19}

Ambulatory Measurement of Skin Temperatures and the Sleep-Wake-Cycle in Women with Vasospastic Syndrome and Controls

\author{
B. Gompper ${ }^{1}$, S. Vollenweider ${ }^{1}$, C. Renz' ${ }^{1}$, E. Van Someren ${ }^{2}$, \\ A. Wirz-Justice1, S. Orgüß ${ }^{1}$ J. Flammer ${ }^{3}$, K. Kräuchi ${ }^{1}$
}

${ }^{1}$ Centre for Chronobiology, Psychiatric University Clinics, Basel, Switzerland; ${ }^{2}$ Netherlands Institute for

Neuroscience and VU Medical Center, Amsterdam, The

Netherlands; ${ }^{3}$ University Eye Clinic, Basel, Switzerland

Introduction: The primary vasospastic syndrome is defined as a vascular dysregulation of blood vessels in otherwise healthy subjects (main symptoms: cold hands and feet). It occurs mostly in young women and is often accompanied by sleep onset insomnia (SOI). In this study, we investigated when vasospasm occurs under normal everyday conditions. In addition we asked whether women having both vasospastic syndrome and SOI (VS) have different internal phase relationships between thermoregulation, sleep midpoint, sleepiness and the light-dark cycle in comparison to controls (CON). Methods: Study subjects were $20 \mathrm{VS}$ and $21 \mathrm{CON}$ women (Age: $25.4 \pm 0.7$ s.e.m., BMI: $21.0 \pm 0.3$ ). Skin temperatures were recorded in a week-long ambulatory protocol using 11 wireless temperature sensors (iButtons; left and right wrist, ankle, calf, thigh, infraclavicular region, sternum; 2.5-min intervals). Purified data $2 \mathrm{~h}$ before and after lights off and lights on, respectively, were analysed, as well as mean 24-hour profiles of raw data (7 days). Subjective items such as sleepiness, sleep times etc. were recorded daily in sleepactivity diaries. Results: Compared with CON, VS showed increased distal vasoconstriction at midday and in the evening, as indicated by lower distal skin temperatures (DIST, mean of hands and feet), feetcalf and distal-proximal skin temperature gradients $(\mathrm{p}<0.05)$. VS revealed distal vasoconstriction before lights off, which lasted longer after lights off than in CON. Sleep onset latency was longer in VS vs. CON (29.2 \pm 4.3 vs. $7.8 \pm 1.1 \mathrm{~min})$, but times of lights off and lights on did not differ. Calculated sleep midpoint was later in VS compared to $\mathrm{CON}(4.68 \pm 0.22$ vs. $4.00 \pm 0.23 \mathrm{~h})$, but no differences were found in sleep duration. Subjective ratings of sleepiness (VAS) showed a phase delay in VS vs. CON $(p<0.02)$. Conclusions: Under everyday conditions VS demonstrate a phase delay in DIST, sleep midpoint and sleepiness with respect to the light-dark cycle compared with CON. Therefore, VS exhibit certain aspects of a chronobiological disorder. Support: Research supported by SNF Grant \#3100A0-102182 to K.K. and the Schwickert-Stiftung.

\section{P20}

\section{Women with Vasospastic Syndrome Exhibit Difficulties Initiating Sleep and Turn Their Experienced Anger Inwards}

\author{
M. von Arb' ${ }^{1}$, B. Gompper', P. Fontana ${ }^{1}$, S. Vollenweider', \\ S. Orgül2, J. Flammer², E. Zemp Stutz ${ }^{3}$, K. Kräuchi ${ }^{1}$ \\ ${ }^{1}$ Centre for Chronobiology, Psychiatric University Clinics, \\ ${ }^{2}$ University Eye Clinic, ${ }^{3}$ Institute of Social and Preventive \\ Medicine, University of Basel, Basel, Switzerland
}

Introduction: Primary vasospastic syndrome (VS) is a functional disorder of vascular regulation in otherwise healthy subjects, mostly in women, with cold hands and feet as leading symptoms. Epidemiological, ambulatory and controlled laboratory studies show evidence for a close relationship between VS and difficulties initiating sleep. Anger/aggression problematic is known to correlate with vascular dysregulation as well as with sleep disturbances. The aim of the study is to elucidate whether women with VS exhibit a higher level of anger/aggression than controls. Methods: 146 women were recruited from participants of a larger survey in a random population sample of Basel-Stadt (Kräuchi et al., APSS, 2005) filling in detailed postal questionnaires on sleep behavior, thermal discomfort, anger and anger expression. Women with VS and controls $(\mathrm{CON})$ were classified using questionnaire-derived scores (feeling of cold hands and feet, finger color changes). Anger/aggression scores were derived from the statetrait-anger-expression-inventory (STAXI) used for dispositional state (S-A), trait anger (T-A), and for anger expression. Anger expression consists of three subscales: Anger-In (A-I), Anger-Out (A-O), and Anger-Control (A-C). Results: In comparison to controls $(\mathrm{N}=84)$, women with VS $(\mathrm{N}=62)$ showed significantly higher VS-scores ( $2.85 \pm 0.06$ vs. $1.60 \pm 0.03$ units) and increased sleep onset latency (SOL; $23.2 \pm 2.3$ vs. $14.7 \pm 1.6 \mathrm{~min})(\mathrm{p}<0.05$, t-test). S-A, T-A, A-O and A-C did not differ between the groups, however, VS showed significantly higher values of A-I than CON $(15.5 \pm 0.6$ vs. $13.7 \pm 0.5$ units $)$. Multivariate backward stepwise regression analysis controlling for BMI, age and smoking revealed high VS-score as the most significant predictor for high A-I. In turn, among these variables, high A-I is the best predictor for long SOL. Conclusions: In this sample, high VSscore is not only associated with long SOL but also with high A-I, indicating a possible role of anger/aggression problematic in the genesis of VS and difficulties initiating sleep. Support: Research supported by the Schwickert-Stiftung and the SNF Grant \#3100A0-102182 to K.K.

\section{Reference}

1. Kräuchi K, Fontana Gasio P, Renz C, Orgül S, Flammer J, Zemp Stutz E: The Basel survey on sleep behavior and vasospastic syndrome: evidence for an association of sleep onset insomnia with peripheral vasoconstriction. Sleep 2005;28(suppl):A236-A237. 


\section{P21}

\section{Sleep Patterns, Work and Strain among Young Students in Hospitality and Tourism}

\section{S. Brand' , B. Hermann'ㄹ, F. Muheim', J. Beck', M. Hatzinger ${ }^{1}$,} E. Holsboer-Trachsler ${ }^{1}$

${ }^{1}$ Psychiatric University Clinics, Depression Research Unit, Basel, ${ }^{2}$ Swiss School of Hospitality and Tourism, Passugg, Switzerland

Objective: Good and sufficient sleep is crucial for a good quality of life. We investigated the associations between sleep patterns, work and strain among students of hospitality and tourism. Methods: 92 students completed psychological and sleep-related questionnaires, and a sleep/work log for one week. Results: Sleeping hours were inversely correlated with working hours. Decreased sleep quality was associated with increased scores of strain, depression and anxiety. Participants with increased working hours were 3.2 times more likely to report heightened insomnia scores than those with lower weekly working hours. Working on weekends was associated with increased strain with family life and peers. Conclusions: In hospitality and tourism, the employees' 'personal costs' for a 24/7 service may be underestimated; unfavourable work schedules are linked with decreased sleep quality, symptoms of depression, anxiety and with social problems.

\section{P22 \\ Electroencephalographic Sleep Profiles, Hypothalamic-Pituitary-Adrenocortical (HPA) Activity, and Association with Psychological/Behavioural Variables in Pre-Schoolers}

\section{Hatzinger ${ }^{1}$, S. Brand ${ }^{1}$, S. Perren ${ }^{2}$, S. Stadelmann², A. von Wyl ${ }^{2}$, K. von Klitzing ${ }^{2}$, E. Holsboer-Trachsler ${ }^{1}$ \\ ${ }^{1}$ Psychiatric University Clinics UPK, Depression Research Unit, ${ }^{2}$ Psychiatric University Clinics UPK, Department of Child and Adolescent Psychiatry, University of Basel, Basel, Switzerland}

Objectives: Sleep regulation and hypothalamic-pituitaryadrenocortical (HPA) system functioning are associated with major psychiatric disorders such as depression. However, most of the data available so far are from studies after the disorder's onset. Therefore, we started a project in pre-school children aiming to objectively investigate sleep, HPA axis function, and psychological/behavioural variables in order to identify risk factors early in development. Here, we report the first cross-sectional investigation in pre-schoolers. Subjects and Methods: For sleep registration, 67 pre-schoolers ( 35 boys and 32 girls) at the age of 5 years underwent EEG-monitoring for one night after an adaption night with mock-registration. EEG analyses were performed according to standard procedures. For baseline HPA activity assessment, saliva samples were collected immediately after waking up, whereas saliva samples before, during and after psychological challenge were used to measure HPA activity under stress conditions. Salivary cortisol was analysed with a commercially available RIA kit. Results: A gender difference was documented in the EEG sleep patterns: Compared to girls, boys showed significantly more REM sleep $(\mathrm{p}<0.05)$. Independent of gender, cluster analysis revealed that children labelled as bad sleepers $(n=27)$ showed significantly increased morning cortisol values compared to normal sleepers. In contrast, children rated as good sleepers displayed the lowest morning cortisol values. Moreover, sleep EEG markers such as an increased number of awakenings after sleep onset, more time in stage 1, 2, and in REM sleep were associated with increased AUC basal cortisol values under stress conditions. Taken together, an association between unfavourable sleep patterns and increased HPA activity under stress conditions could be observed. With respect to psychological/behavioural variables, an increased sleep efficiency was significantly correlated with low degrees of impulsivity $(\mathrm{r}=0.31 ; \mathrm{p}<0.05)$, bullying and social inhibition $(\mathrm{r}=0.26$, $\mathrm{p}<0.05$ ); in contrast, a decreased sleep efficiency was significantly correlated with denial, that is, with the psychosocial attitude to avoid to perceive social conflicts. Conclusions: Our results show (1) that bad sleep is associated with heightened HPA system activity already in pre-school children, and (2) that unfavourable sleep patterns are related to more difficult behavioural/psychological dimensions. The longitudinal follow up will demonstrate if these biological abnormalities are predictive for the onset of clinically relevant psychiatric problems such as depression.

P23

Serotonergic and Dopaminergic Stimulation in Relation to Approach-Avoidance Behavior in Patients with Anxiety Disorders and Borderline Personality Disorder

\author{
U. Hemmeter ${ }^{1}$, B. Kundermann'1, B. Nekwasil', A. Thum', \\ Th. Schneyer ${ }^{1}$, M. Bender², M. Huber ${ }^{1}$, M. Giesler', \\ J.C. Krieg \\ ${ }^{1}$ University of Marburg, Clinic of Psychiatry and \\ Psychotherapy, Marburg, ${ }^{2}$ ZSP Rehbergpark Herborn, \\ Germany
}

Introduction: Patients with Borderline Personality disorder (BPD) and with anxiety disorders (AD) contrast in personality traits and behavior. According to Gray impulsivity and anxiety are independent personality traits characterized by a different susceptibility for stimuli of reward and punishment. In addition, these traits have been related to serotonergic and dopaminergic neurotransmission. Methods: To further elucidate the relationship between anxiety and impulsivity, performance and neurobiology a study in 30 patients with BPD and AD, which includes the parallel assessment of personality, behavioral performance and neuroendocrine responsiveness to serotonergic and dopaminergic stimulation has been conducted. All patients had to complete different personality questionnaires (e.g. NEO FFI, TCI) and underwent an approach-avoidance paradigm in which reward and punishment were systematically varied. Furthermore, a serotonergic (oral appl. citalopram $20 \mathrm{mg}$ vs. placebo) and dopaminergic (oral appl. bromocriptin $1.25 \mathrm{mg}$ ) test assessing cortisol and prolactin was performed. Results: Cortisol and prolactin were significantly more stimulated by citalopram in BPD compared to AD. No differences between groups were observed in the dopaminergic 
stimulation. The approach-avoidance paradigm revealed a significant better performance in the condition, in which reward could be easily achieved for BPD compared to AD. No differences were found in the 'difficult' reward condition and conditions of punishment. Citalopram induced cortisol-secretion was significantly related to performance in the reward condition. Discussion: Patients with AD und BPD clearly differ in distinct dimensions of personality and show a different serotonergic neurotransmission according to citalopram stimulation. The observed difference in serotonergic neurotransmission may be related to personality pattern and performance.

\section{P24 \\ Prediction in Psychiatry: Stress and Sleep in Kindergarten Children}

M. Hatzinger ${ }^{1}$, S. Brand ${ }^{1}$, S. Perren ${ }^{2}$, S. Stadelmann ${ }^{3}$, A. von Wy/ ${ }^{3}$, K. von Klitzing ${ }^{4}$, E. Holsboer-Trachsler ${ }^{1}$

${ }^{1}$ Psychiatric University Clinics UPK, Depression Research Unit, Basel, '2Jacobs Center for Productive Youth

Development, University of Zurich, ${ }^{3}$ Psychiatric University Clinics UPK, Department of Child and Adolescent

Psychiatry, University of Basel, Switzerland; ${ }^{4}$ Department of Child and Adolescent Psychiatry, Psychotherapy, and

Psychosomatics University of Leipzig, Germany

Within recent years the hypothalamic-pituitary-adrenocortical (HPA) system and sleep regulation have reached considerable attention in psychiatry. E.g. profound alterations of these neurobiological systems in psychiatric disorders like depression were reported in adults. However, the issue if these abnormalities precede the development of psychiatric disorders and may contribute to the identification of high-risk subjects early in life still warrants clarification. In order to assess HPA system functioning and sleep regulation as possible candidates for prediction of psychiatric disorders a cross-sectional and longitudinal study was started in children during the developmental period at the turn from a home-based context to a peerdominated environment. Here, we report the first cross-sectional investigation's results. 102 children (age $4.91 \pm 0.44$ years, 53 boys, 49 girls) at the beginning of Kindergarten were enrolled in a first cross-sectional examination of (1) basal and stress-challenged HPA system activity assessed by saliva cortisol measurements and (2) sleep regulation investigated by actigraphy over 7 days. Basal HPA axis regulation was assessed by morning cortisol after awakening, whereas stress-elicited hormonal responses were measured during the application of a standardized psychological stress test appropriate at this age (McArthur Story Stem Battery, MSSB). In parallel, children's psychological and psychosocial assessment was carried out. Baseline HPA system activity was significantly increased in girls when compared to boys $(p<0.001)$. Furthermore, high morning cortisol secretors predicted a high hormonal release during the MSSB with - again - females showing higher hormonal responses than males $(\mathrm{p}<0.01)$. Additionally, heightened HPA system activity was related to unfavorable psychological values in a gender specific manner: whereas boys had an increased hyperactivity/impulsivity $(Z=-2.2, p<0.05)$ and more conduct/externalizing problems $(Z=-2.81, \mathrm{p}<0.01)$, girls experienced more emotional/internalizing problems $(Z=-2.3$, $\mathrm{p}<0.05)$. Sleep recordings revealed severe disturbances in up to
$15 \%$ of the investigated children with no gender difference. Particularly these subjects had significantly increased morning cortisol values when compared to good sleepers $(p<0.05)$. Moreover, they showed significantly elevated scores of dysregulated aggression and of a worse family climate $(\mathrm{p}<0.05)$. The present results in children point to a possible occurrence of neurobiological abnormalities already early in development. These alterations may be gender specific already at the age of 5 years. Prospective long-term follow up of these identified subjects will clarify if these markers are predictive for the onset of psychiatric disorders.

Supported by the Swiss NSF, $\mathrm{N}^{\circ} 32-68193.02$ (M.H.) and $\mathrm{N}^{\circ} 32-66778.01$ (K.v.K.)

\section{P25}

\section{A Randomized Controlled Pilot Trial of Methylphenidate and Cognitive-Behavioral Group Therapy for Cocaine Dependence in Heroin Prescription}

\author{
K.M. Dürsteler-MacFarland ${ }^{1}$, C. Bürki² ${ }^{2}$ J. Strasser ${ }^{1}$, \\ S. Petitjean ${ }^{1}$, D. Ladewig ${ }^{1}$, G.A. Wiesbeck ${ }^{1}$ \\ ${ }^{1}$ Department of Psychiatry, University of Basle and \\ ${ }^{2}$ Department of Psychiatry, University of Bern, Switzerland
}

Cocaine dependence has proved difficult to treat, whether occurring alone or in combination with opiate dependence. No intervention has been demonstrated to be uniquely effective. Patients might benefit most from combined pharmaco- and psychotherapeutic interventions. The present study sought to evaluate the feasibility, tolerability and efficacy of methylphenidate (MP) and cognitive-behavioral group therapy (CBGT) for cocaine dependence in patients prescribed heroin. Sixty-two cocaine-dependent, heroin-maintained patients participated in a dual-site, double-blind, placebo-controlled pilot trial with 4 treatment conditions. Participants were randomly assigned to receive MP or placebo each of which combined with either CBGT or treatment as usual for twelve weeks. MP $30 \mathrm{mg}$ and placebo in identical capsules were administered onsite twice daily under supervision. Manual-guided CBGT consisted of 12 weekly sessions (in groups of 5-7 patients). Primary outcome measures were cocaine-free urines, retention in pharmacologic treatment, and adverse effects. Urine cocaine screens were performed thrice weekly. Subjective measures were weekly assessed. Seventy-one percent of participants completed the trial. There were no significant differences in patient characteristics across treatment groups. MP was well tolerated with similar retention rates compared to placebo. No serious adverse effects occurred. Cocaine use persisted in all groups. Using GEE modeling the longitudinal data showed no significant change in cocaine-free urines. In conclusion, MP did not provide an advantage over placebo in reducing cocaine use. In contrast to positive results from the USA obtained in other samples, this study does not support a role for CBGT for treating cocaine dependence in heroin-maintained patients. There were no signs of additive benefits of MP and CBGT. 


\section{P26}

\section{Controlled Study on the Combined Effects of Alcohol and Tobacco on Testosterone in Alcohol-Dependent Men}

\section{G.A. Wiesbeck, U. Gerhard, M. Walter}

Department of Psychiatry, University of Basle, Switzerland

More than $90 \%$ of alcohol-dependent men are heavy smokers. However, little is known about the combined effects of both substances. This study is the first one to examine the impact of drinking and smoking on plasma testosterone in alcohol-dependent men. A total of 51 alcohol-dependent men with ICD-10 alcohol dependence were investigated. Their free testosterone in plasma was measured on the day of admission $\left(\mathrm{t}_{1}\right)$, after successful detoxification $\left(\mathrm{t}_{2}\right)$ and after six weeks of sobriety $\left(t_{3}\right)$. They were compared to 43 age-matched healthy male control subjects. While the testosterone level of alcoholics did not differ from controls at the onset of withdrawal $\left(t_{1}\right)$, it was significantly higher for alcoholics after 6 weeks of sobriety $\left(t_{3}\right)$. Higher alcohol consumption and higher tobacco use before detoxification led to higher levels of testosterone before $\left(t_{1}\right)$ and after withdrawal $\left(t_{2}\right)$. These data give evidence to the hypothesis that the combined effect of alcohol and tobacco is cumulative. High levels of alcohol and tobacco consumption are associated with high levels of testosterone before and after alcohol withdrawal.

\section{P27}

Sleep Behavior of the Swiss Population: The Prevalence and the Day-Time Consequences of Insomnia

\section{A. Delini-Stula1, R. Bischof', E. Holsboer-Trachsler ${ }^{2}$ \\ ${ }^{1} \mathrm{ADI}$ International Institute for Advancement of Drug Development, ${ }^{2}$ Universitäre Psychiatrische Klinik, Basel, Switzerland}

Introduction: High prevalence of sleep disturbances and particularly their chronic course are today a recognized medical and socioeconomic problem but still underestimated in the practice. A survey of the sleep behavior of the Swiss population was therefore performed with the aim to assess the need for improvement of the diagnostics and the treatment. Method: A specifically constructed questionnaire was addressed to a random sample of subjects (men and women $\geq 18$ years old). The interviews were carried out by telephone (computerized CATI system) by a specialized institution. No incentives were given to the surveyed subjects. Statistics: The data were descriptively analyzed. The significance of results was ascertained, if appropriate, with non-parametric statistical tests. Results: The sample consisted of men and women $(\mathrm{N}=1002$, mean age $39.5 \pm 15.18)$ from all Swiss regions. The analysis showed that about $1 / 3 \mathrm{rd}(31.3 \%)$ of the total population and about equal proportion of man and women suffers from some degree of insomnia, defined by stringent DSM-IV criteria. The highest prevalence was found in retired $(48 \%)$ and widowed subjects $(>65 \%)$, but of concern was also rather high prevalence $(33.4 \%)$ of insomnia in younger adults (18-24 years old).
Day-time consequences of insomnia were marked and clearly severitydependent. They affected between $25-100 \%$ of insomniacs. They included impairments of physical and mental health. The most common were overfatigue, mood- (depression, loss of joy) and affect(anxiety, tension, irritability) changes combined with reduced performance capacity and disturbances of cognition (memory loss, concentration and attention deficits). Unsatisfactory quality of life $(>70 \%)$, sexual problems and impaired social life were reported by more than 2/3rd of subjects. Conclusion: These results of this analysis show that, like in other Western countries, insomnia is also a medical and socio-economic problem in Switzerland that needs an increased attention in the practice.

\section{P28}

\section{Association of COMT Single Nucleotide Polymorphisms with Loudness Dependence of Auditory Evoked Potentials (LDAEP)}

\author{
W. Kawoh/2, I. Giegling ${ }^{3}$, P. Mavrogiorgou', O. Pogare/l', \\ C. Mulert ${ }^{3}$, U. Hegerl', D. Rujescu ${ }^{3}$, G. Juckel $^{1}$ \\ 1 Department of Psychiatry, Ruhr-University Bochum, \\ Germany; ${ }^{2}$ Department of Psychiatry, Psychiatric \\ University Hospital, Zurich, Switzerland; ${ }^{3}$ Department of \\ Psychiatry, University of Munich, Germany
}

The loudness dependence of auditory evoked potential (LDAEP) estimated with the source magnitude of the tangential dipole provides a well established and reliable measure of the central serotonergic activity. Other parameters of the serotonin metabolism such as the concentration of the metabolite 5-hydroxy-indolacetic-acid (5-HIAA) in the cerebro-spinal fluid of the serotonin-transport in thombocytes fall short for mirroring functional aspects of the central serotonergic system. A higher serotonergic activity has been reported as important for the aetiology of schizophrenic disorders. A diminished LDAEP as an epiphenomenon of a higher serotonergic activity has been verified in schizophrenic patients. Additionally, a dopaminergic influence on the LDAEP has been hypothesized as well. After $D_{1} / D_{2}$ and $D_{3}$ dopamine receptor agonism had no effect on the LDAEP, in this study the relationship between dopaminergic function and LDAEP is investigated from another starting-point: the association of Catechol O-methyltransferase (COMT) single nucleotide polymorphisms rs165599, Val158Met (rs4680) and rs737865 and LDAEP is tested. COMT is a catecholamine degrading enzyme, functional polymorphisms of the COMT gene result in attenuated dopamine catabolism and thus in higher levels of dopamine. They are discussed to confer risk for psychotic disorders. 95 healthy subjects of German descent (41 males, 54 females) were included and received electrophysiological recording and blood drawing for genotyping. Interestingly, single marker analysis showed weak associations with loudness dependence for two of the three tested SNPs, sliding haplotype analysis after dropping rare haplotypes showed a similar pattern for the same SNPs with an association trend with lower loudness dependence scores. Haplotype analysis with all SNPs showed association with loudness dependence. These findings support the hypothesis that the LDAEP is also influenced by dopaminergic transmission. 
P29

\section{Therapeutic Strategies in the Treatment of Depressed Patients in the Practice: The Example of Mirtazapine}

A. Delini-Stula1, R. Bischof 1 , B. Scherrer ${ }^{1}$, M. Kokic ${ }^{2}$

${ }^{1} \mathrm{ADI}$ International Institute for Advancement of Drug Development, Basel, ${ }^{2}$ Organon AG, Pfäffikon, Switzerland

Introduction: Even though many algorithms and guidelines for the treatment of depressed patients exist, the empiry shows that in the every-day practice the physicians are seldom compliant with them. But, there are hardly studies, which have systematically analyzed factors influencing or determining physician's decision about the treatment schedules and dosing regimes of antidepressant in the practice. We have in this sense explored the results of an observational study with mirtazapine, an established antidepressant drug. The final aim of the analysis was to find out which treatment strategy provided optimum benefit to the patient. Methods: A sample for analysis consisted of depressed, male and female patients $\geq 18$ years old $(\mathrm{N}=487)$ attending private practice setting in Switzerland. They were treated for 8 weeks with mirtazapine in an open, prospective, non-interventional, observation study. Both the selection of patients for treatment and the dosing regime of mirtazapine were in this study entirely left to the discretion of the investigators. Demographic and other variables related to treatment and treatment outcome, pre- and co-medication as well as adverse events were recorded at baseline, after about 4 weeks and at the end of the trial. The interactions and relationship between the dosing schedules and a number of possible interfering variables were analyzed, among others, by means of a multivariate factor analysis. Results: The results revealed three principal treatment strategies: (a) variable dosing (increasing-decreasing), (b) stepwise increasing doses and (c) fixed dose treatment throughout. The applied doses varied from 15 to maximum $90 \mathrm{mg} /$ day. The factors best explaining the selection of these treatment strategies (from low to high doses) were, surprisingly enough, not the features of depression $(p=0.99)$, but severity of anxiety (positive correlation with high starting doses, $\mathrm{p}=0.0005$ ), severity of somatic symptoms (negative correlation, $\mathrm{p}=$ 0.0001 ) and age (negative correlation if combined with high somatisation, $\mathrm{p}=0.0001$ ). The results further showed that by comparison to other treatment schemes, fixed dose regime and moderate to high initial dose ( $\geq 30 \mathrm{mg} /$ day) provide maximum benefit to patients regarding both efficacy and safety of treatment. The poorest response was found in patients starting at dose below $30 \mathrm{mg}$ /day and changing the dose during the treatment. The initiation of treatment with high doses ( $\geq 45 \mathrm{~g} /$ day) proved to be of no disadvantage for the patient and, particularly, was not associated with an increased incidence of adverse effects. Conclusion: The results of this study showed that, contrary to frequent habits in the practice, the most effective treatment strategy with mirtazapine, promising the best outcome for the patients, is to initiate the therapy with higher doses $(\geq 30 \mathrm{mg}$ /day) and to maintain the dose throughout the treatment period.
P30

\section{Performance in Face Recognition Task Differentiates Fregoli from Capgras}

S. Walther, A. Federspiel, H. Horn, M. Wirth, W. Strik, T. Müller

University Hospital of Psychiatry, Bern, Switzerland

Introduction: Schizophrenics show deficits in face identity recognition and facial emotion perception. Patients with delusional misidentification syndromes have problems integrating identity and affective information to a face. Several DMS are quite frequent in schizophrenia. Still research on the topic is incomplete. Method: 20 schizophrenics and 21 control subjects were presented with faces to memorize. Afterwards they were tested for recognition accuracy and speed. During the recognition task, participants were presented with famous faces, unknown faces and the previously learned faces. They had to indicate, whether they know the face or not. Results: Schizophrenics with delusional misidentification syndromes (DMS) have longer reaction times $(p<0.001)$ than controls or patients without DMS. Patients with positive DMS (Fregoli) display longer reaction times than patients with negative DMS (Capgras). In terms of accuracy, Capgras leads to poor accuracy for newly learned faces (28\%) and famous $(22 \%)$ faces, while their performance is outstanding for unknown faces (96\%). Schizophrenics with Fregoli's syndrome have better accuracy for previously learned faces $(53 \%)$, comparable accuracy for famous faces (31\%) and worse accuracy for unknown faces (51\%). Conclusions: Schizophrenics with DMS are more disabled in a face recognition task. The pattern of disturbances differentiates between Capgras and Fregoli syndromes, suggesting different neurophysiological pathways to both disorders. 
P31

\section{Encoding Deficit During Face Processing Within the Fusiform Face Area in} Schizophrenia

S. Walther ${ }^{1}$, A. Federspiel' ${ }^{1}$, H. Horn ${ }^{1}$, P. Bianchi ${ }^{1}$, R. Wiest ${ }^{2}$, M. Wirth ${ }^{1}$,W. Strik ${ }^{1}$, T. Müller ${ }^{1}$

${ }^{1}$ University Hospital of Psychiatry, ${ }^{2}$ Institute of Diagnostic and Interventional Neuroradiology, Inselspital, University of Bern, Bern, Switzerland

Background: Face processing is crucial to social interaction, but is impaired in schizophrenics, who experience delays in face recognition, difficulties identifying others, and misperceptions of affective content. The right fusiform face area plays an important role in the early stages of human face processing and thus may be affected in schizophrenia. The aim of the study was therefore to investigate whether face processing deficits are related to dysfunctions of the right fusiform face area in schizophrenics compared to controls. Methods: In a rapid, event-related fMRI design, we investigated the encoding of new faces, as well as the recognition of newly learned, famous, and unfamiliar faces, in 13 schizophrenics and 21 healthy controls. We applied region of interest analysis to each individual's right fusiform face area and tested for group differences. Results: Controls displayed more BOLD activation during the memorization of faces that were later successfully recognized. In schizophrenics, this effect was not observed. During the recognition task, schizophrenics exhibited lower BOLD responses, less accuracy, and longer reaction times to famous and unfamiliar faces. Discussion: Our results support the hypothesis that impaired face processing in schizophrenia is related to early stage deficits during the encoding and immediate recognition of faces. 


\section{Neuropsychobiology}

Abraham, T. P1

Anders, D. P18

Aston, J. P2, P3, P4, P5, V1

Aubry, J.-M. V4

Bach, D.R. P9

Bars, D. P1

Bassetti, C.L. S4

Baysang, G. V8

Beck, J. P21

Bender, M. P23

Bianchi, P. P31

Bischof, R. P27, P29

Blatter, K. P16

Borgwardt, S. P2, V1

Bovet, P. P8

Brand, S. P21, P22, P24, V6

Brandeis, D. P12

Bromundt, V. P15, P17

Brown, S.A. V7

Büchel, N. P5

Buclin, T. P8

Bull, N. P3, P4

Bürki, C. P25

Cajochen, C. P16, P17, V7

Chitnis, X. V1

Conus, P. P8

Cuenod, M. P8, V2

Czech, C. V8

Dammann, G. P17

Dayer, A. P14

Dazzan, P. V1

Delini-Stula, A. P27, P29

Deppen, P. P8

De Quervain, D.J.-F. P18

Dierks, T. P7, P12, P13, V3

Do, K.Q. P8, V2

Drewe, M. P4, P5, V1

D'Souza, M. V1

Dürsteler-MacFarland, K.M. P25, V9

Eckert, A. V7, V8
Numbers refer to abstract numbers
Faber, P.L. P11

Federspiel, A. P6, P7, P12, P13, P30, P31

Flammer, J. P19, P20

Flux, B. V5

Fontana, P. P20

Fuhr, P. P1

Gerhard, U. P26

Giegling, I. P28

Giesler, M. P23, V5

Gompper, B. P19, P20

Götz, J. V8

Gschwandtner, U. P1, P2, P4, P5, V1

Gysin, R. V2

Haag, A. V5

Haller, S. V1

Hatzinger, M. P21, P22, P24, $\mathrm{S} 2, \mathrm{~V} 6$

Hegerl, U. P28

Hemmeter, U. P23, V5

Hermann, B. P21

Holsboer-Trachsler, E. P21, P22, P24, P27, V6

Horn, H. P6, P7, P30, P31

Huber, M. P23

Hubl, D. V3

Izakovic, J. V7

Juckel, G. P28

Kawohl, W. P28

Kiefer, C. P13

Kill, A. P15

Klee, P. V5

Knoblauch, V. P16

Knyazeva, M. P8

Kochi, K. P11

Koenig, T. P7, P10, P11, P12, V3

Kokic, M. P29

Kräuchi, K. P18, P19, P20

Krieg, J.C. P23, V5
Kundermann, B. P23, V5

Kyburz, S. P17

Ladewig, D. P25

Landolt, H.-P. S3

Laverrièr, A. V4

Lavoie, S. P8, V2

Lehmann, C. P12

Lehmann, D. P11

Luethi, M. V6

McGuire, P.K. V1

Mavrogiorgou, P. P28

Meier, B. P7

Meier, F. V7, V8

Meuli, R. P8

Muheim, F. P21

Mulert, C. P28

Müller, S. V9

Müller, T. P6, P7, P30, P31

Müller-Spahn, F. V8

Münch, M. P16

Murray, M.M. P8

Nekwasil, B. P23

Orgül, S. P19, P20

Ozmen, L. V8

Pagani, L. V7

Perren, S. P22, P24

Petitjean, S. P25, V9

Pflueger, M. P2

Pflüger, M. P4, P5, V1

Pogarell, O. P28

Pollmächer, T. S1

Python, A. V4

Radü, E.W. V1

Rao, H. P13

Razafimandimby, A. P7

Rechsteiner, E. P3, V1

Renz, C. P19

Rhein, V. V8
Riecher-Rössler, A. P1, P2, P3, P4, P5, V1

Rujescu, D. P28

Savaskan, E. V8

Scherrer, B. P29

Schneyer, Th. P23

Schulz, P. V4

Semenin, V. P1

Senn, W. P10

Solida, A. P8

Späti, J. P16

Stadelmann, S. P22, P24

Steimer, T. V4

Stein, M. P7, P12

Steullet, P. V2

Stieglitz, R.-D. P2, P4, V1

Stoppe, G. P15

Strasser, J. P25

Strik, W. P6, P7, P10, P11, P12, P30, P31, V3

Thum, A. P23, V5

Van Someren, E. P19

van Swam, C. V3

Vollenweider, S. P18, P19, P20

von Arb, M. P20

von Klitzing, K. P22, P24

von Planta, A. V6

von Wyl, A. P22, P24

Waldmeier, L. V7

Walter, M. P26

Walther, S. P30, P31

Waltinger, T. P11

Wang, J.J. P13

Wiesbeck, G.A. P25, P26, V9

Wiest, R. P6, P12, P31

Wirth, M. P6, P7, P30, P31

Wirz-Justice, A. P15, P17, P18, P19, V7

Wurst, F.M. V9

Zemp Stutz, E. P20

\section{KARGER}

(C) 2007 S. Karger AG, Basel

Fax +41613061234

E-Mail karger@karger.ch

www.karger.com

Accessible online at:

www.karger.com/nps 